\title{
Orientational order and morphology of clusters of self-assembled Janus swimmers
}

\author{
Francisco Alarcon, ${ }^{1}$ Eloy Navarro-Argemí, ${ }^{2,3}$ Chantal Valeriani, ${ }^{1,{ }^{*}}$ and Ignacio Pagonabarraga ${ }^{2,3,4}$ \\ ${ }^{1}$ Departamento de Estructura de la Materia, Física Térmica y Electrónica and GISC, Universidad Complutense \\ de Madrid 28040 Madrid, Spain \\ ${ }^{2}$ Departament de Física de la Matèria Condensada, Universitat de Barcelona, C. Martí Franqués 1, 08028 Barcelona, Spain \\ ${ }^{3}$ University of Barcelona Institute of Complex Systems (UBICS), Universitat de Barcelona, 08028 Barcelona, Spain \\ ${ }^{4}$ CECAM, Centre Européen de Calcul Atomique et Moléculaire, École Polytechnique Fédérale de Lasuanne, Batochime, Avenue Forel 2, \\ 1015 Lausanne, Switzerland
}

(Received 1 January 2019; published 6 June 2019)

\begin{abstract}
Due to the combined effect of anisotropic interactions and activity, Janus swimmers are capable to selfassemble in a wide variety of structures, many more than their equilibrium counterpart. This might lead to the development of novel active materials capable of performing tasks without any central control. Their potential application in designing such materials endows trying to understand the fundamental mechanism in which these swimmers self-assemble. In the present work, we study a quasi-two-dimensional semidilute suspensions of two classes of amphiphilic spherical swimmers whose direction of motion can be tuned: either swimmers propelling in the direction of the hydrophobic patch or swimmers propelling in the opposite direction (toward the hydrophilic side). In both systems we have systematically tuned swimmers' hydrophobic strength and signature and observed that the anisotropic interactions, characterized by the angular attractive potential and its interaction range, in competition with the active stress, pointing toward or against the attractive patch gives rise to a rich aggregation phenomenology.
\end{abstract}

DOI: 10.1103/PhysRevE.99.062602

\section{INTRODUCTION}

Since the late 2000s, the pioneer experiments by Dombrowski and Cisneros [1,2] inspired the numerical work aimed at understanding the influence of hydrodynamics in the formation of coherent structures of micro-organisms, simulated as spherical squirmers [3] in three dimensional (3D) [4,5] and quasi-2D [6]. The features of a 3D squirmer suspension have been numerically characterized using the smoothed profile method [7-9] and lattice Boltzmann (LB), either in bulk [10] or in response to a steady Couette flow [11]. While Evans et al. [12] used Stokesian dynamics to detect the instability of the isotropic state in a 3D suspension of weak pullers, Delmotte and coworkers [13] applied a force-coupling method to investigate, by large-scale simulations, the emergence of a polar order in a 3D squirmer suspension.

More recently, experiments by Ref. [14-18] have inspired numerical simulations of $2 \mathrm{D}$ and quasi-2D suspensions of squirmers interacting via a repulsive potential.

When a suspension (simulated by means of multiparticle collision dynamics) was confined between parallel walls (quasi-2D geometry) [19], active particles were forming clusters whose size depended on the system's concentration with the largest being made of pullers. The suspension prepared in such confined geometry would undergo phase separation into a dilute and a dense phase [20], differently to the 2D suspension of disklike squirmers [21], where hydrodynamics suppressed phase separation.

\footnotetext{
*cvaleriani@ucm.es
}

Inspired by the self-assembly observed in experiments with either bacteria [16] or active colloids [17] and by numerical works on 2D dilute suspensions of active Brownian particles (ABP) interacting via isotropic short-range attractions [22-25], we have studied a semidiluted quasi-2D suspensions of attractive squirmers [26] to unravel the role played by hydrodynamics in suspensions of attractive active particles.

Most of the nowadays-synthetic active colloids are colloids partially coated with Pt. These colloids self-propel by a catalytic reaction of $\mathrm{H}_{2} \mathrm{O}_{2}$ and $\mathrm{O}_{2}$ taking place at the $\mathrm{Pt}$ surface. This mechanism of colloid's propulsion is due to phoresis. On the one side, properly modeling the phoretic interactions can be quite complicated [27-32], even though there are experiments, simulations, and theories that consider them relevant for particles' propulsion. On the other side, which interactions dominate in these systems are still a matter of debate [33].

From the experimental point of view, several studies have focused on understanding the effect of hydrophobicity in catalytic Janus colloids. Modifying the surface of the particles, the authors of Ref. [34] found that particles with an hydrophobic patch would move faster than the ones with an hydrophilic patch. Similarly, Gao et al. [35], studied selfassembly of amphiphilic particles made with hydrophobic octadecyltrichlorosilane- (OTS) modified silica microspheres capped with a catalytic Pt hemisphere patch. Additionally, Yan et al. [36] have recently reported different collective states of active dipolar Janus colloids, whose motion is originated by an induced-charge electrophoresis. These colloids self-assemble in different morphologies, ranging from gas, swarms, chains, and clusters by modifying both the charge 
imbalance of the colloids and the electric field frequency. Moreover, in Ref. [36], the authors were able to numerically reproduce all observed states by means of an overdamped molecular dynamics simulation whose particles were interacting via directed imbalanced interactions.

Motivated by these two experimental works [35,36], simulations of a dilute quasi-2D suspension of ABP interacting via anisotropic amphiphilic Janus interactions have been reported in Ref. [37]. To the best of our knowledge, this is the first numerical attempt to unravel the collective behavior of amphiphilic active Janus particles, taking into account their anisotropic nature. In Ref. [37] both the effect of interaction directionality and the propulsion speed have been used to control the physical properties of the assembled active aggregates. One might suggest that these two parameters could be easily tuned also in experiments, given that the concentration of a cationic surfactant could be used to reverse the propulsion's direction and adding $\mathrm{pH}$ neutral salts could be used to control the propulsion speed [38].

In order to establish the relevance of hydrodynamics in this suspension, we study the collective behavior of quasi2D suspensions of spherical squirmers interacting via an anisotropic Janus potential, considering different interaction ranges, interaction strengths, and hydrodynamic signatures. When clusters appear, we characterize their morphology as a function of the above-mentioned features.

The objective of this manuscript is the analysis of minimal models that contain the essential ingredients of activity and steric interactions for heterogeneous particles. The scenarios we identify can be clearly correlated with the competition between self-propulsion, stress generation, and attraction among particles. It is true that phoresis is more complex and can also involve long-range interactions among the active elements. Our results serve as a benchmarking scenario if phoresis is controlled by near field effects. In this sense, the systematic analysis we have carried out provides a valuable contribution in this general perspective. In a more specific perspective, this study also helps to understand the effect of the hydrodynamic interactions in the collective dynamics of active Janus colloids, inspired by experiments showing flow fields around a single catalytic Janus sphere [39].

The manuscript is organized as follows. In Sec. II we present the anisotropic interaction model, the squirmer model, the lattice Boltzmann methodology and simulations details. In Sec. III we describe the tools used to characterize the Janus squirmers. In Sec. IV we present all results and discuss our conclusions in Sec. V.

\section{SIMULATION DETAILS}

In this work, we have studied a quasi-two-dimensional dilute suspension of squirmers [40-42] interacting via a Janus anisotropic potential similar to the one used in Ref. [37] for ABP. In order to model the Janus swimmers, we have introduced a pair potential $V\left(r_{i j}, \theta_{i}, \theta_{j}\right)$ which depends on the distance between two squirmers $\left(r_{i j}\right)$ and their attractive patches' orientations,

$$
V\left(r_{i j}, \theta_{i}, \theta_{j}\right)=V_{\text {rep }}\left(r_{i j}\right)+V_{\text {att }}\left(r_{i j}\right) \phi\left(\theta_{i}, \theta_{j}\right),
$$

where $\theta_{i}$ is the angle between the patch unit vector $\vec{p}_{i}$ and the interparticle vector $\vec{r}_{j i}=\vec{r}_{j}-\vec{r}_{i}$; and $\theta_{j}$ the angle between $\vec{p}_{j}$ and $\vec{r}_{i j}=-\vec{r}_{j i}$.

$V_{\text {rep }}\left(r_{i j}\right)$ represents a short-range soft repulsion [43] (to avoid overlapping) given by

$$
\begin{aligned}
& V_{\text {rep }}\left(r_{s}\right) \\
& \quad= \begin{cases}\epsilon_{s}\left\{\left(\frac{\sigma_{s}}{r_{s}}\right)^{v}-\left(\frac{\sigma_{s}}{h_{0}}\right)^{v}\left[1-\frac{\left(r_{s}-h_{0}\right) v}{h_{0}}\right]\right\} & 0<r_{s} \leqslant h_{0} \\
0 & \text { otherwise, }\end{cases}
\end{aligned}
$$

where $\epsilon_{s}=1.5$ is the energy scale, $v=2, r_{s} \equiv r_{i j}-\sigma$ is the interparticle distance (with $r_{s}>0$ to ensure that particles never overlap), $\sigma_{s}=0.5$ is the characteristic separation length between particles, and $h_{0}$ the distance at which the potential is shifted to ensure its continuity at $r_{s}=h_{0}{ }^{1}$

The attractive potential consists of a radial $V_{\text {att }}\left(r_{i j}\right)$ and an angular $\phi\left(\theta_{i}, \theta_{j}\right)$ contribution. Concerning the radial part of the potential, we have considered two different truncated Lennard-Jones: one long $\left(r_{c}=2.5 \sigma\right)$ and another short $\left(r_{c}=\right.$ $1.5 \sigma)$ range. While the following equation:

$$
\begin{aligned}
& V_{\text {att }}\left(r_{i j}\right) \\
& \quad= \begin{cases}4 \epsilon\left[\left(\frac{\sigma}{r_{i j}}\right)^{12}-\left(\frac{\sigma}{r_{i j}}\right)^{6}\right] & \left(2^{1 / 6} \sigma\right)<r_{i j}<r_{c}, \\
-\epsilon & r_{i j}<\left(2^{1 / 6} \sigma\right), \\
0 & \text { otherwise, }\end{cases}
\end{aligned}
$$

represents the former, it has been modified for the latter by adding a smooth transition function, so that the potential $V_{\text {att }}$ and its derivative vanish continuously as $r_{i j}$ approaches $r_{c}$ [44] (see Appendix A).

Therefore, whenever $\left(2.2^{1 / 6} \sigma\right)<r_{i j}<r_{c}$

$$
\begin{aligned}
V_{\text {att }}\left(r_{i j}\right)= & 4 \epsilon\left[\left(\frac{\sigma}{r_{i j}}\right)^{12}-\left(\frac{\sigma}{r_{i j}}\right)^{6}\right] \\
& +4 \epsilon\left[6\left(\frac{\sigma}{r_{c}}\right)^{12}-3\left(\frac{\sigma}{r_{c}}\right)^{6}\right]\left(\frac{r_{i j}}{r_{c}}\right)^{2} \\
& -4 \epsilon\left[7\left(\frac{\sigma}{r_{c}}\right)^{12}+4\left(\frac{\sigma}{r_{c}}\right)^{6}\right],
\end{aligned}
$$

whereas for $r_{i j}<\left(2.2^{1 / 6} \sigma\right) V_{\text {att }}=-0.305 \epsilon$ and $V_{\text {att }}=0$ otherwise.

Inspired by the work of Hong et al. [45] for amphiphilic colloidal spheres, the anisotropic interaction is controlled by the angular contribution of the attractive potential,

$$
\begin{aligned}
\phi & \left(\theta_{i}, \theta_{j}\right) \\
& = \begin{cases}\cos \theta_{i} \cos \theta_{j} & \left(\hat{p}_{i} \cdot \hat{\mu}_{j i}\right)>0 \cap\left(\hat{p}_{j} \cdot \hat{\mu}_{i j}\right)>0, \\
0 & \text { otherwise } .\end{cases}
\end{aligned}
$$

where $\hat{\mu}_{i j}=\vec{r}_{i j} / r_{i j}$ is the unit vector joining the squirmers' center of mass.

\footnotetext{
${ }^{1}$ Note that separation $h_{0}$ is relatively short, $h_{0}<\sigma_{s}$, and its value depends on the location of the minimum of the attractive potential.
} 
(a)

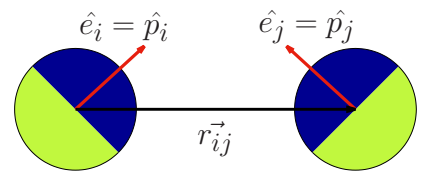

WP

(b)

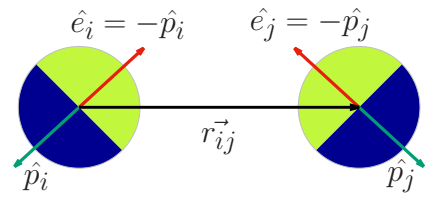

AP

FIG. 1. (a) WP Janus swimmers. (b) AP Janus swimmers. The attractive patch is represented in blue (dark gray), and the nonattractive patch is in green (light gray). The swimming direction $\hat{e}$ is the red arrow and direction of the attractive patch $\hat{p}$ is the green one. The center-to-center distance between two swimmers $\overrightarrow{r_{i j}}$ is the black vector.

This anisotropic potential ensures a nonvanishing torque when $\left(\hat{p}_{i} \cdot \hat{\mu}_{j i}\right)>0$ and $\left(\hat{p}_{j} \cdot \hat{\mu}_{i j}\right)>0$ (a more detailed analysis is discussed in Appendix B).

Within our model, each Janus particle self-propels along a direction $\hat{e}_{i}$ rigidly bounded to the particle. As in Ref. [37], we study two types of active Janus particles depending of the orientation of $\hat{e}_{i}$ with respect to $\hat{p}_{i}$. When the propulsion direction $\left(\hat{e}_{i}\right.$, red arrow in Fig. 1, left panel) is pointing toward the attractive patch $\hat{p}_{i}$ (red arrow in Fig. 1, left panel), swimmers are propelling "with the patch" (WP). When the propulsion direction ( $\hat{e}_{i}$, red arrow in Fig. 1, right panel) is pointing against the attractive patch $-\hat{p}_{i}$ (green arrow in Fig. 1, right panel), swimmers are propelling "against the patch" (AP).

A spherical Janus squirmer of radius $R_{p}$ is characterized by the tangential surface slip velocity,

$$
\left.\mathbf{u}\right|_{R_{p}}=\left[B_{1} \sin \theta+B_{2} \sin \theta \cos \theta\right] \boldsymbol{\tau},
$$

where $\tau$ is a unit vector tangential to the particle surface, $B_{1}$ quantifies the asymptotic self-propelling speed of an isolated squirmer $\left(v_{s}=\frac{2}{3} B_{1}\right)[46,47]$, and $B_{2}$ is proportional to the active stress generated on the surrounding fluid. The ratio between active stress and self-propelling velocity, $\beta=B_{2} / B_{1}$ [3], quantifies the active state of the squirmer and its interaction with the fluid. A positive value of $\beta$ corresponds to the case when thrust is generated in front of the squirmer's body (puller); a negative value of $\beta$ to the case when thrust is generated at the back (pusher) [4] and $\beta=0$ corresponds to a neutral swimmer (see Fig. 2). In our simulations we disregard thermal fluctuations; thus velocity fluctuations are simply induced by the particles' activity, where $B_{2}$ can be understood as an effective temperature.

In order to characterize how hydrodynamics affects the assembly of a dilute suspension of Janus swimmers, we model the embedding solvent using a lattice Boltzmann (LB) method with a D3Q19 three-dimensional lattice $[48,49]$, implemented in a highly parallelized code [50] that exploit the excellent scalability of LB on supercomputing facilities. Spherical squirmers (with a diameter $\sigma$ of 4.6 lattice nodes [51]) are individually resolved, imposing a modified bounceback rule on the one-particle velocity distribution functions to nodes crossing the solid particle's boundary (including the slip velocity to impose the squirming motion [52,53]). The total force and torque the fluid exerts on the particle is obtained by imposing that the total momentum exchange between the particle and the fluid nodes vanishes (as a result of the modified bounce-back). While accounting for all forces acting on each squirmer allows to update them dynamically, the torque exerted by the fluid determines the change of the squirmer's self-propulsion direction.

The simulated system consists of a suspension of $N=888$ spherical squirmers at $\phi=0.10$ (where $\phi=\frac{\pi}{4} \rho \sigma^{2}$ and $\rho=$ $\left.N / L^{2}\right)$ in a quasi-two-dimensional geometry with periodic boundary conditions $(L \times L \times k \sigma$, with $k=5$ and $L \approx 83 \sigma)$ needed to capture three-dimensional hydrodynamics effects, whereas both colloids' position and orientation are confined to move in 2D. Additionally, we have carried out bigger simulations with $N=3552$ squirmers at the same packing fraction and $L \approx 167 \sigma$, in order to prove that results are not due to finite-size effects, especially cases with large cluster sizes.

As in Ref. [26], we quantify the competition between attractive and self-propelling forces via the dimensionless parameter

$$
\xi=\frac{F_{d}}{F_{\mathrm{att}}},
$$

where $F_{d}=6 \pi \eta R_{p} v_{s}$ is the friction force associated to the squirmer self-propulsion and $F_{\text {att }}$ is the absolute value of

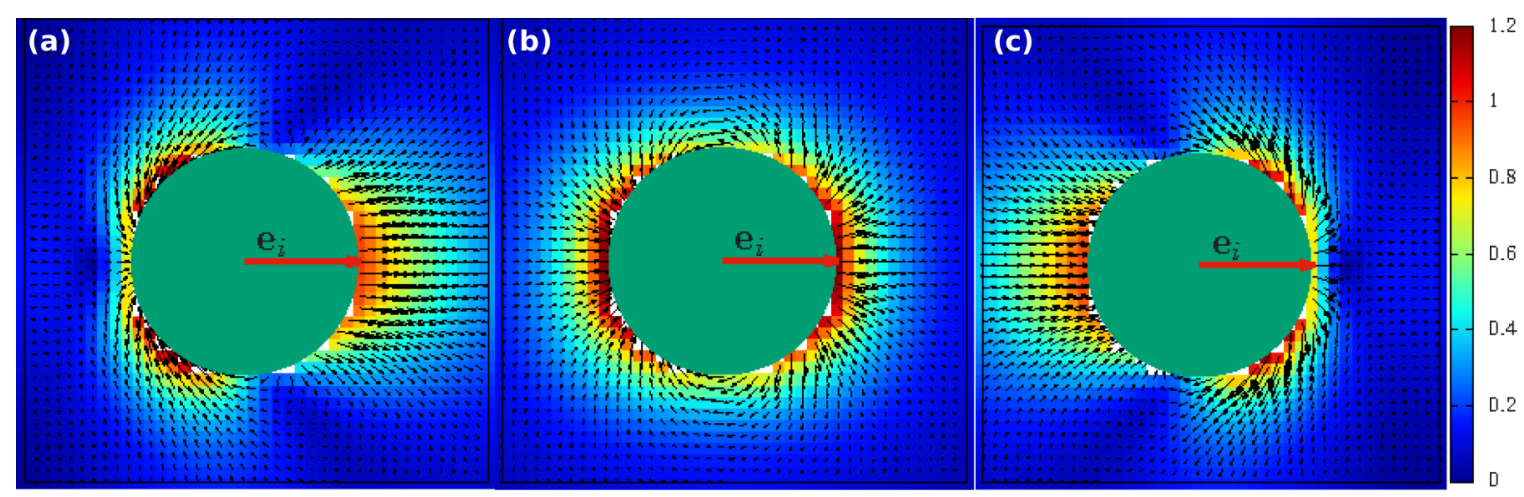

FIG. 2. XY projection of a squirmer at $\beta=-1$ (pusher, left panel), $\beta=0$ (neutral, middle panel), and $\beta=1$ (puller, right panel). The red arrow represents the swimming direction, the black arrows the velocity directions of the fluid, the color code the speed of the fluid normalized by the Stokes velocity $\left(v_{s}=2 / 3 B_{1}\right)$. Velocities are computed in the squirmer frame of reference. 
the attractive force at its minimum, corresponding to $r=$ $(26 / 7)^{1 / 6} \sigma=1.245 \sigma$ for the long-range potential in Eq. (3) and to $r=1.239 \sigma$ for the short-range potential in Eq. (4). On the one hand, $\xi$ controls the competition between two mechanisms: the self-propulsion and the interaction strength. On the other hand, the mechanisms that control the reorientation of the particles are the active stress given by the $B_{2}$ squirmer parameter and the orientational dependence of the potential which is modulated by the range and the strength of the interaction. Given that a squirmer travels its own size in a time $\tau=\frac{\sigma}{v_{s}}$, we perform simulations from 1450 up to $3000 \tau$.

Once the system reaches steady state, in a time window between 1000 and $2000 \tau$, we carry out a systematic analysis of the dynamics of the squirmer suspension, considering $\xi$ from 0.1 to 10 and $\beta$ from -3 to 3 .

\section{ANALYSIS TOOLS}

In order to be able to establish the effect played by the anisotropy of the interaction between swimmers, we follow the procedure in Ref. [26] and study the degree of aggregation as a function of $\beta$ and $\xi$. Whenever the system forms a steadystate cluster distribution, we explore the clusters morphology by computing their mean size, the cluster-size distribution, their radius of gyration, and both the polar and nematic order parameter.

When clusters are present, we identify them following a distance criterion: At each time step, two particles belong to the same cluster of size $s$ whenever their distance is smaller than $r_{\mathrm{cl}}=1.75 \sigma$ [first minimum of the $g(r)$, see Appendix C].

To calculate the cluster-size distribution $f(s)$ we apply a criterion based on [54]: (i) We arbitrarily subdivide the range of $s$ values into intervals $\Delta s_{i}=\left(s_{i, \max }-s_{i, \min }\right)$, where $n_{i}^{t}$ is the total number of clusters within each interval $\Delta s$; (ii) we assign the value $n_{i}=n_{i}^{t} / \Delta s_{i}$ to every $s$ within $\Delta s_{i}$ and compute the fraction of clusters of size $s$ as $f(s)=n_{i} / N_{c}$, where $N_{c}=\sum_{i} n_{i} \Delta s_{i}$ is the total number of clusters.

To characterize the clusters' morphology, we compute the radius of gyration,

$$
R_{g}(s)=\sqrt{\sum_{i, j=1}^{s} \frac{\left(\vec{r}_{i}-\vec{r}_{j}\right)^{2}}{2 s}},
$$

the average polar order parameter for each cluster size $s$,

$$
P(s)=\left|\frac{\sum_{i=1}^{s} \hat{e}_{i}}{s}\right|,
$$

the tensor order parameter (for a 2D system [55]),

$$
Q_{h k}(t)=\frac{1}{N} \sum_{i=1}^{N}\left[2 e_{i h}(t) e_{i k}(t)-\delta_{h k}\right],
$$

(with $h$ and $k$ equal to $x, y$ and $N$ the total number of squirmers), and the nematic order parameter $\lambda(t)$ being the largest eigenvalue of $Q_{h k}$ [56].

We have also computed both global nematic and global polar order parameters, substituting $s$ with $N$ in the global polar order parameter [Eq. (9)]. Therefore, the polar order in steady state is $P_{\infty}=P(t \gg 0)$ while the nematic order in steady state is $\lambda_{\infty}=\lambda(t \gg 0)$.
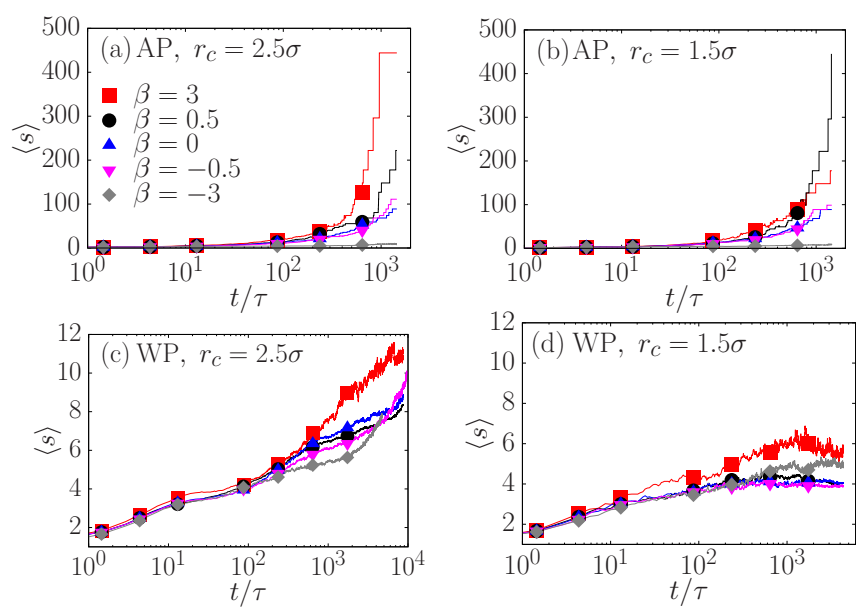

FIG. 3. Time evolutions of the mean cluster size for squirmers when $\xi=0.1$ (pullers: red squares and black circles; neutral: blue up-triangles; pushers: pink down-triangles and gray diamonds). (a) AP squirmers with $r_{c}=2.5 \sigma$ and (b) $r_{c}=1.5 \sigma$, (c) WP squirmers with $r_{c}=2.5 \sigma$, and (d) $r_{c}=1.5 \sigma$. Note the different scale on the $y$ axis.

\section{RESULTS}

\section{A. Mean cluster size}

We start with computing the mean cluster size $\langle s\rangle$ as a function of time for different values of $\beta$ and $\xi$. This allows us to distinguish coarsening from clustering, establishing, in the latter case, when the system reaches a steady state.

Figure 3 represents the mean cluster size as a function of time for suspensions where attraction dominates over propulsion $(\xi=0.1)$ of AP squirmers (interacting via longrange [Fig. 3(a)] or short-range [Fig. 3(b)] attraction) and of WP squirmers (interacting via long-range [Fig. 3(c)] or short-range [Fig. 3(d)] attraction). In all AP suspensions the system coarsen, within the simulated time window as shown by the continuously growing mean cluster size, with a speed dependent on $\beta$. Pullers coarsening faster than pushers, apart from the system with $\beta=-3$ where particles form clusters independently on the interaction range.

WP suspensions only coarsen when interactions are long range [Fig. 3(c)] with a speed dependent on $\beta$ and pullers coarsening faster than pushers, even though coarsening is much slower than in the AP case. In [Fig. 3(c)], the coarsening dynamics is so slow that $\langle s\rangle<10$ even when $t>1000 \tau$ (it is worth noting that we have never observed a steady state despite having run up to $6 \times 10^{6} \mathrm{LB}$ time steps, corresponding to $\sim 8700 \tau$ ). Comparing Fig. 3(c) and Fig. 3(d), we conclude that the attraction range affects aggregation, given that when the interaction is short range [Fig. 3(d)], coarsening is suppressed and $\langle s\rangle$ reaches a steady state at long $t$ for all values of $\beta$. A more detailed analysis is given in Appendix D for the case of the WP system with $\xi=0.1$.

Figure 4 represents the mean cluster size as a function of time for suspensions where attraction competes with propulsion $(\xi=1)$ of AP squirmers (interacting via long-range [Fig. 4(a)] or short-range [Fig. 4(b)] attraction) and of WP squirmers (interacting via long-range [Fig. 4(c)] or shortrange [Fig. 4(d)] attraction). 

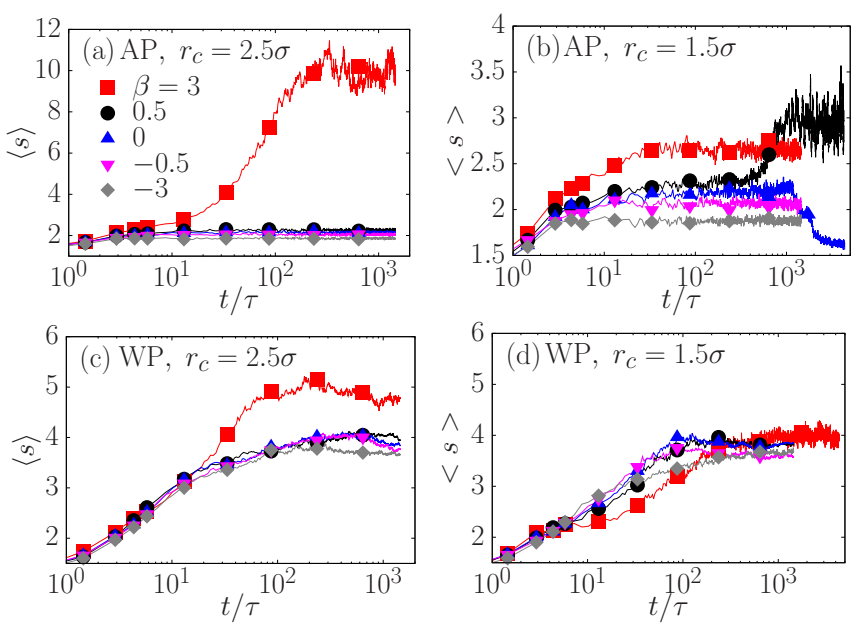

FIG. 4. Time evolutions of the mean cluster size for squirmers when $\xi=1$ (pullers: red squares and black circles; neutral: blue uptriangles; pushers: pink down-triangles and gray diamonds). (a) AP squirmers with $r_{c}=2.5 \sigma$ and (b) $r_{c}=1.5 \sigma$, (c) WP squirmers with $r_{c}=2.5 \sigma$ and (d) $r_{c}=1.5 \sigma$. Note the different scale on the $y$ axis.

AP squirmers interacting via long-range attractions [Fig. 4(a)] quickly reach a steady state characterized by $\langle s\rangle \approx$ 2 except for $\beta=3$, where $\langle s\rangle \approx 10$ [red curve in pFig. 4(a)]. Whereas when dealing with AP squirmers interacting via short-range attractions [Fig. 4(b)], pushers reach a steady state quite quickly with $\langle s\rangle \approx 2$ as well as pullers with $\beta=3$ (with a slightly larger mean cluster size of $\langle s\rangle \approx 2.5$ ). Interestingly, the mean cluster size for weak pullers (black curve in Fig. 4(b)] first develops a metastable state at short times $[t \in(10,500) \tau]$ and then another one for longer times $(t>$ $1000 \tau)$ : the latter due to the formation of dynamic clusters with polar order. Similarly, when $\beta=0$ the decay in $\langle s\rangle$ at long time corresponds to monomers aligning and moving in the same direction.
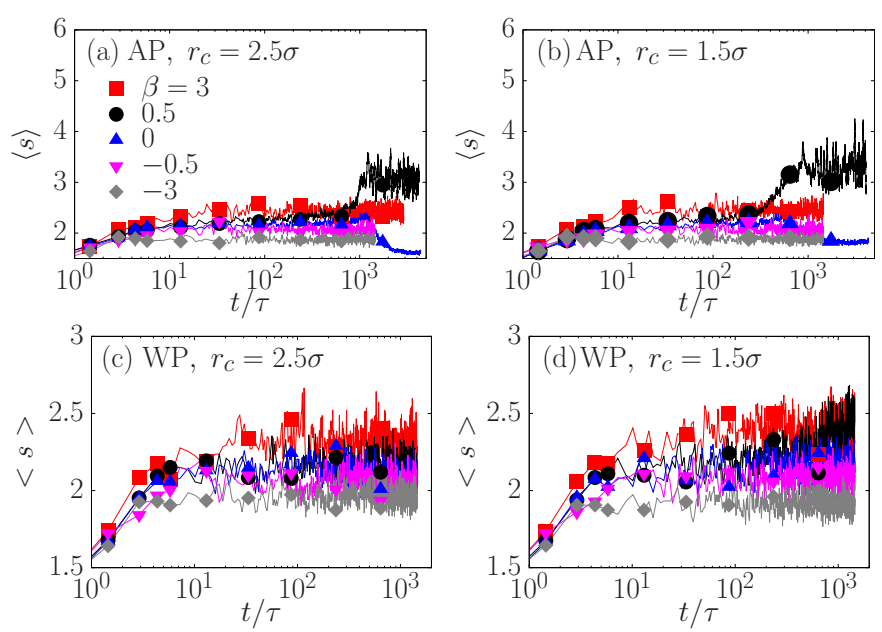

FIG. 5. Time evolutions of the mean cluster size for squirmers when $\xi=10$ (pullers: red squares and black circles; neutral: blue uptriangles; pushers: pink down-triangles and gray diamonds). (a) AP squirmers with $r_{c}=2.5 \sigma$ and (b) $r_{c}=1.5 \sigma$, (c) WP squirmers with $r_{c}=2.5 \sigma$, and (d) $r_{c}=1.5 \sigma$. Note the different scale on the $y$ axis.
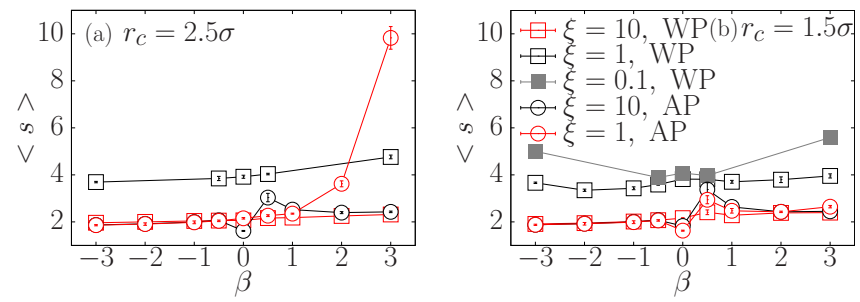

FIG. 6. Mean cluster size for suspensions with (a) $r_{c}=2.5 \sigma$ and (b) $r_{c}=1.5 \sigma$ in steady state for WP $(\xi=10, \xi=1$, and $\xi=0.1$, square symbols) and $\mathrm{AP}(\xi=10$ and $\xi=1$, circular symbols $)$

WP squirmers with $\beta<3$ relatively quickly reach steady state with clusters with an average of four particles [Fig. 4(c)]. Whereas pullers with $\beta=3$ reach a slightly higher mean cluster size $\langle s\rangle \sim 5$ : Such increase in the cluster size is observed only for this interaction range, since WP squirmers with short-range attractions [Fig. 4(d)] develop a mean cluster size of approximately four particles, independently on their hydrodynamic signature.

Figure 5 represents the mean cluster size as a function of time for suspensions where propulsion dominates over attraction $(\xi=10)$ of AP squirmers (interacting via longrange [Fig. 5(a)] or short-range [Fig. 5(b)] attraction) and of WP squirmers (interacting via long-range [Fig. 5(c)] or short-range [Fig. 5(d)] attraction).

When self-propulsion dominates over attraction, squirmers in general form smaller clusters (never larger than four particles on average). In either AP suspensions, the mean cluster size for weak pullers [black circles in Figs. 5(a) and 5(b)] is larger than the one for any other $\beta$, fluctuating around a value of 3 for long times. In the case of neutral squirmers and large $\xi$ and after a long time $(t>1000 \tau)$, particles in the suspension become strongly aligned and most of the clusters are monomers, for both interaction ranges.

When dealing with WP squirmers and high $\xi$, we have observed a small value of the mean cluster size (around 2), which indicates that particles form dimers on average. A similar behavior is observed for WP squirmers with shortrange interactions, with slightly larger fluctuations in the mean cluster size for weak pullers at long times.

Having established the conditions needed for clustering to appear, we represent in Fig. 6 the mean cluster size as a function of $\beta$ for $r_{c}=2.5 \sigma$ [Fig. 6(a)] and $r_{c}=1.5 \sigma$ [Fig. 6(b)]. ${ }^{2}$

As shown in Fig. 6, when $\xi=1 \mathrm{WP}$ swimmers mostly form tetramers and trimers, while WP swimmers with $\xi=10$ form dimers. The clusters observed for WP squirmers have an average size rather insensitive to the squirmer hydrodynamic signature for these two interaction strength $\xi=\{1,10\}$ and the two interaction ranges studied here.

For lower values of $\xi$, the dependence on $\beta$ becomes more evident for WP squirmers with $\xi=0.1$ [gray squares on Fig. 6(b)]: For extreme values of $\beta$ (either pushers or pullers)

${ }^{2}$ The WP system with $\xi=0.1$, where clustering is observed [Fig. 3(d)], is studied in more detail in Appendix D. 
the clusters on average form pentamers, whereas neutral and weak pullers or weak pushers are mostly form tetramers.

AP squirmers with $r_{c}=2.5 \sigma$ [Fig. 6(a)] show that the mean cluster size increases as $\beta$ increases when $\xi=1$, whereas it does not change significantly when $\xi=10$ or when the interaction range is shorter [circular symbols Fig. 6(b)].

As summary, on the one side, comparing the two panels in Fig. 6 , when $\xi=1$ and 10 , the mean cluster size $\langle s\rangle$ for WP squirmers with interaction range of $r_{c}=1.5 \sigma$ behaves in the same way as the one with a larger cutoff $\left(r_{c}=2.5 \sigma\right)$. Therefore, when attraction does not dominate, the mean cluster size for WP squirmers is independent on the interaction range. On the other side, the mean cluster size $\langle s\rangle$ for AP squirmers with $\xi=1$ and $r_{c}=1.5 \sigma$ [red circles in Fig. 6(b)] does not behave in the same way than when $r_{c}=2.5 \sigma$ [red circles in Fig. 6(a)], the former showing a systematic lower value of $\langle s\rangle$ for all $\beta$ while the latter a monotonic increase of the cluster size for pullers. Finally, AP squirmers with $\xi=10$ (black circles in Fig. 6) show the same behavior varying $\beta$, independently on the attraction range. Both cases show a small peak for weak pullers, and a minimum at $\beta=0$.

\section{B. Coarsening, clustering, and aligned states}

Figure 7 displays the different states and types of collective motion or self-assembly that WP and AP Janus squirmers form as $\beta$ and $\xi$ vary. This way of classifying our simulations allow us to observe in a better way all the results.

We have identified seven different cases: three gas systems, three clustering systems, and one coarsening state. All the different cases show different types of cluster morphologies and/or the particles' alignment. The emerging structures result to be sensitive to the patch direction (WP or AP), the hydrodynamic signature $\beta$ and the interaction range $r_{c}$ and strength $\xi$. The three gas systems are characterized by the different particles' alignment: isotropic (with particles randomly swimming), polar (with most of the particles swimming in the same direction), and nematic (with approximately half of the total amount of particles swimming in one direction and the other half in the opposite direction). The three clustering systems can be classified as finite-size dynamic clusters, chains, and trimers. In the coarsening case all particles form a unique macroscopic aggregate.

AP Janus squirmers coarsen into a single cluster for $\xi \approx$ 0.1 [see Fig. 7(a)] and self-organize into finite-size clusters for $\xi \approx 1$ [see Fig. 7(b)] (larger values of $\beta$ favoring the formation of larger finite-size clusters, accelerating coarsening). When $\xi=10$, AP squirmers either form a polar ordered gas [see Fig. 7(c), for weak positive stresslets, $0<\beta<1$ ] or an isotropic gas (for AP pushers and pullers with $\beta>1$ ). WP Janus squirmers have a strong tendency to form small micelles of three or four particles pointing at each other. For $\xi \approx 0.1$ these micelles coalesce and end up forming chains, which in most of the cases coexist with smaller micelles [see Fig. 7(d)]. At $\xi=1$ the competition with the active stress $(\beta)$ gives rise to trimers and tetramers avoiding the formation of larger chains of particles [see Fig. 7(e)]. If activity dominates $(\xi=10)$ and $|\beta|<1$, even though a polar order is not favored due to the anisotropic interactions, then we still observe a global nematic order: A significant portion of particles move
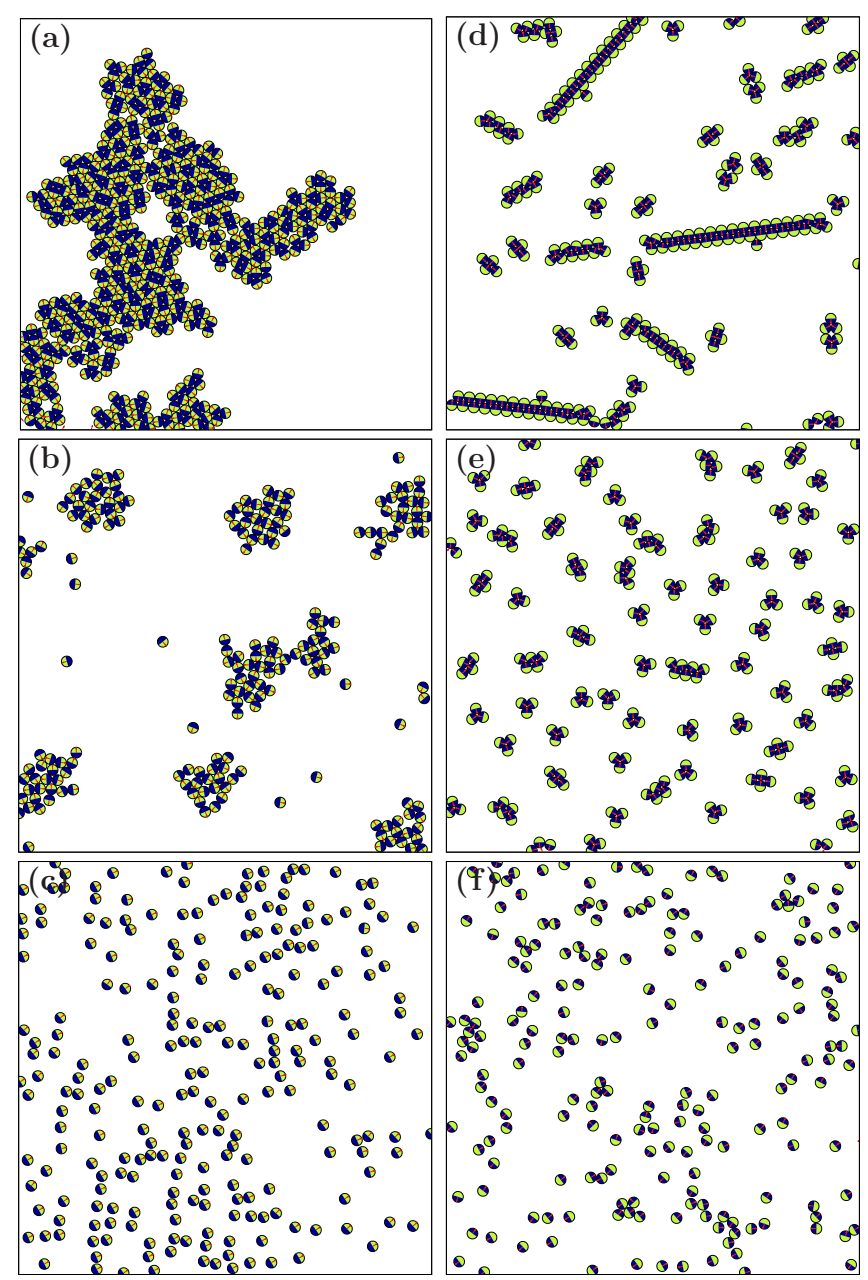

FIG. 7. Snapshots of different types of self-assembly for $r_{c}=$ $2.5 \sigma$. The attractive hemisphere is represented in blue, the repulsive in green and the fixed orientation vector is shown in red. Panels (a), (b), and (c) are AP squirmer suspensions with $\xi=\{0.1,1,10\}$, respectively, while panels (d), (e), and (f) are WP squirmer suspensions with $\xi=\{0.1,1,10\}$, respectively. (a) Coarsening with $\beta=3$. (b) Clustering with $\beta=3$. (c) Gas system with polar order (polar gas) with $\beta=0$. (d) Chains system with $\beta=0$. (e) Trimers state with $\beta=-3$. (f) Gas case with nematic order (nematic gas) with $\beta=0$.

in one direction and the rest in the opposite one [see Fig. 7(f)]. For other values of $\beta$, we observe an isotropic system. In the Supplemental Material [57] we have included the movies of the six simulations represented in Fig. 7 to better capture both the dynamical and morphological features reported in this paper.

\section{Cluster size distribution}

Additionally to the mean cluster size, we calculate the cluster size distribution (CSD) to study the statistics of the different cluster sizes found it for Janus swimmers, computing the CSD for $\xi=1$ and $\xi=10$ and as a function of $\beta$, the direction of the attractive patch and the interaction range. The case when attraction dominates with respect to self-propulsion $(\xi=0.1)$ for AP squirmers is analyzed in Appendix D. Results for $\xi=1$ are presented in Fig. 8 and for $\xi=10$ in Fig. 9 . 

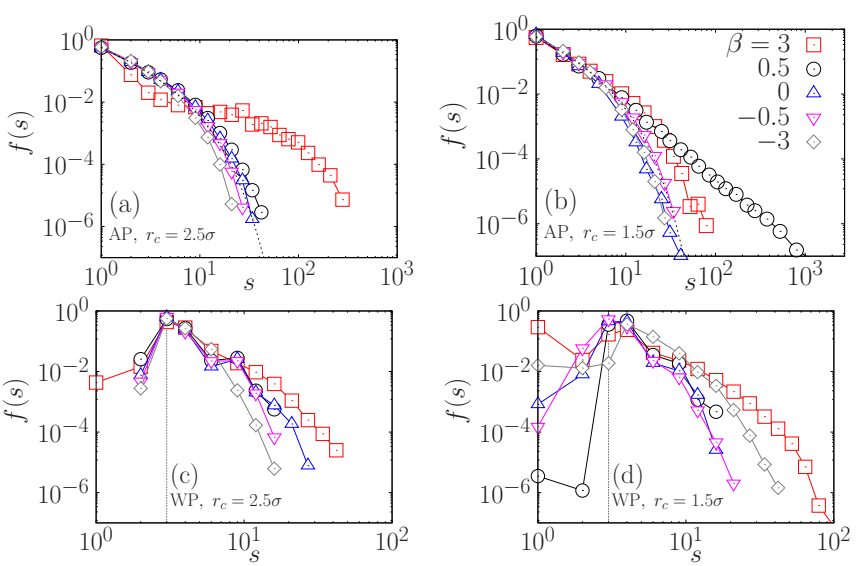

FIG. 8. CSD for $\xi=1$. AP squirmers (a) with $r_{c}=2.5 \sigma$ and (b) $r_{c}=1.5 \sigma$. WP squirmers (c) with $r_{c}=2.5 \sigma$ and (d) $r_{c}=1.5 \sigma$. Gray dashed line in (a) and (b) represents the analytical function for a CSD given by Eq. (11) with $B=0$, plotted as guide to the eye, with $s_{0}=4$, $\gamma_{0}=5 / 4$. In (c) and (d) a vertical dashed line is shown as a reference for $s=3$ (trimers).

Following previous studies [26], where CSD was calculated, we use the same analytical function as a reference,

$$
\frac{f(s)}{f(1)}=A \frac{\exp \left[-(s-1) / s_{0}\right]}{s^{\gamma_{0}}}+B \frac{\exp \left[-(s-1) / z_{0}\right]}{s^{-\gamma_{0}}},
$$

with $\gamma_{0}, s_{0}, z_{0}$, and $B$ constants such that $A=1-B$.

Figure 8(a) represents the CSDs of AP squirmers for $\xi=1$ and $r_{c}=2.5 \sigma$. The characteristic distribution width grows with $\beta$ until $\beta<3$. For all the explored parameters, monomers always represent the largest contribution. For large-enough values of $\beta, \beta=3$, a second peak emerges for cluster sizes involving a few tens of particles (around 25 for the system parameters explored). Accordingly, CSDs with $\beta<3$ can be described by Eq. (11), with $B=0$ while the $\operatorname{CSD}$ for $\beta=3$ requires a nonvanishing value of $B$ in
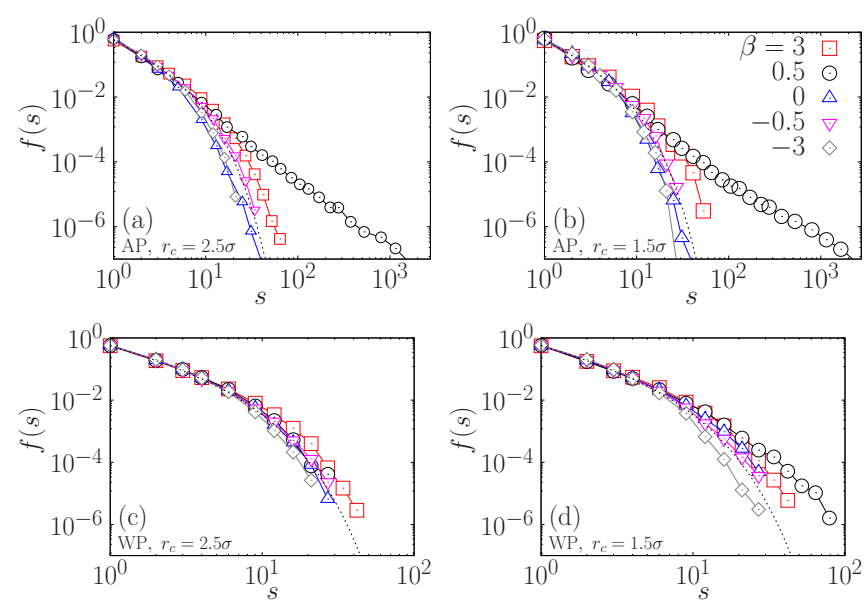

FIG. 9. CSD for $\xi=10$. AP squirmers (a) with $r_{c}=2.5 \sigma$ and (b) $r_{c}=1.5 \sigma$. WP squirmers (c) with $r_{c}=2.5 \sigma$ and (d) $r_{c}=1.5 \sigma$. Gray dashed line in (a) and (b) represents the analytical function for a CSD given by Eq. (11) with $B=0$, plotted as guide to the eye, with $s_{0}=4, \gamma_{0}=5 / 4$.
Eq. (11) to capture the observed maximum. The width of the CSDs is measured by the value of the exponential tail parameter $s_{0}$; thus pushers CSDs have smaller $s_{0}$, whereas the corresponding $s_{0}$ for pullers is larger until $\beta=3$. At larger values of $\beta$ the behavior changes due to the appearance of a second peak in the CSDs.

Figure 8(b) represents the CSDs of AP squirmers for $\xi=1$ and $r_{c}=1.5 \sigma$. CSDs of AP pushers and neutral squirmers follow the analytical behavior of a power law with an exponential tail [Eq. (11) with $B=0$ : gray dashed line] with the same width. The squirmers with $\beta=3$ present a CSD wider than the pushers, whereas weak pullers CSD follows a power-law behavior instead of the power law with an exponential tail [Eq. (11)] which represents clusters of all sizes; such effect is not observed when interaction range is $2.5 \sigma$.

Figure 8(c) represents the CSDs of WP squirmers for $\xi=1$ and $r_{c}=2.5 \sigma$. A peak appears between trimers and tetramers, while the characteristic CSD width increases with the active stress $\beta$. Interestingly, when $\beta<3$ monomers are not observed. Strong WP pushers are characterized by a suspension dominated by trimers, while strong WP pullers form a polydisperse suspension of monomers, dimers, trimers, and even chains of tens of particles.

Figure 8(d) represents the CSDs of WP squirmers for $\xi=1$ and $r_{c}=1.5 \sigma$, with CSDs presenting a peak between trimers and tetramers. In this case, the CSD width depends on the active stress magnitude rather than in its sign: The higher the value of $|\beta|$, the wider the distribution (with distributions wider than the ones with longer interactions range $r_{c}=2.5 \sigma$, and with a higher number of monomers especially when $\beta=3$ ).

Therefore, CSDs present relevant differences depending on whether squirmers are AP- or WP-like and on the interaction range of the potential. The CSDs for AP squirmers decay monotonously as a function of cluster size, with a large tail corresponding to cases in which there is the formation of finite-size aggregates. On the contrary, CSDs for WP usually display a peak for trimers. Moreover, the interaction range affects how the CSDs width depends on the hydrodynamic signature: While for large interaction range, strong AP pullers develop a wider distribution than any other $\beta$, for a shorter interaction range weak pullers develop a power-law distribution.

In contrast, neither the range of interaction among WP squirmers nor the hydrodynamic signature affect the shape of the CSDs, since CSDs with a peak on trimers is present in all cases, with the widest distribution appearing for the stronger WP or AP pullers.

Figure 9(a) represents the CSDs of AP squirmers with $\xi=10$ and $r_{c}=2.5 \sigma$. The corresponding distributions are monotonically decreasing, particularly for $\beta=0.5$ a powerlaw distribution emerges while in the other cases, the CSD can be approximated by Eq. (11) with $B \neq 0$.

Figure 9(b) displays AP squirmers' CSDs for $\xi=10$ and $r_{c}=1.5 \sigma$. The CSDs are essentially the same as in the previous case with longer interaction range for pushers and $\beta=0$, while for pullers the CSDs are lightly wider.

Figure 9(c) displays the CSDs for WP squirmers for $\xi=$ 10 and $r_{c}=2.5 \sigma$. In this case, hydrodynamics does not significantly affect the CSD, since the CSDs are all monotonically decreasing. Although $\beta=3$ is slightly wider and $\beta=-3$ is 

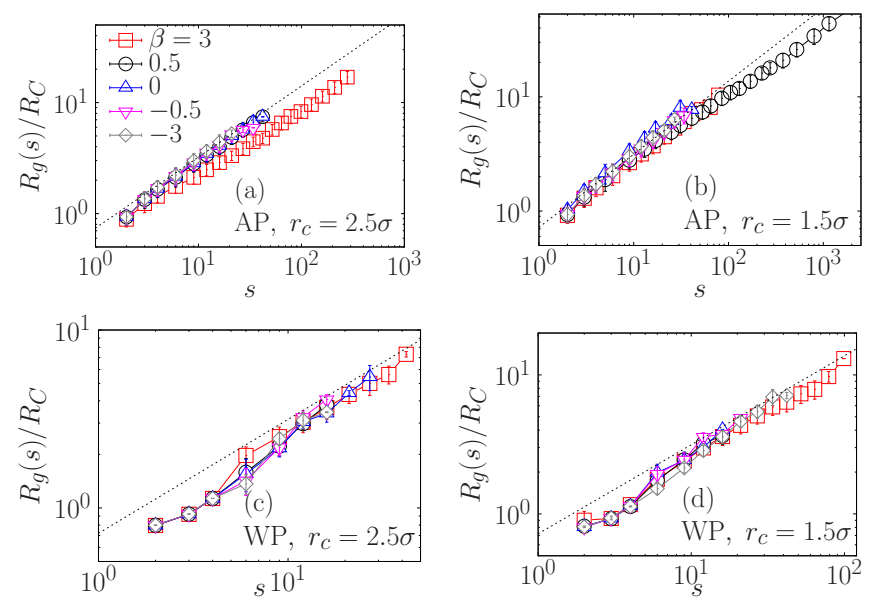

FIG. 10. Radius of gyration normalized by the particle radius as a function of the cluster size for suspensions with $\xi=1$. Top panels are AP squirmers with (a) $r_{c}=2.5 \sigma$ and (b) $r_{c}=1.5 \sigma$, whereas bottom panels are WP squirmers with (c) $r_{c}=2.5 \sigma$ and (d) $r_{c}=$ $1.5 \sigma$. Gray dashed lines in all panels are the function $R_{g}=K s^{0.64}$.

slightly narrower than the others, the distributions are similar among them, with approximately the same width.

Since activity dominates over the interaction strength, we can infer that this independence on $\beta$ is due to two main effects: on the one hand, the anisotropy of the potential and, on the other hand, the range of the interaction. Since in Fig. 9(d) for WP squirmers and $\xi=10$ and $r_{c}=1.5 \sigma$, the CSDs have indeed a different width depending on the hydrodynamic signature, corresponding $\beta=0.5$ to the widest distribution. When activity dominates over the interaction strength, we can infer that CSDs depend mainly on hydrodynamics, but the interaction range plays an important role, too, mainly for AP and WP pullers.

\section{Radius of gyration}

The next feature we compute to study the morphology of the clusters is their radius of gyration $\operatorname{Rg}(s)$ [Eq. (8)], as a function of the cluster size $s$. Figure 10(a) displays the radius of gyration $R_{g}(s)$ of AP squirmers with $\xi=1$ and $r_{c}=2.5 \sigma$. We observe that $R_{g} \sim s^{0.64}$ for $\beta<3$, while for $\beta=3$ [red squares in Fig. 10(a)], $R_{g}$ starts following the same power law before a crossover to a lower value of $R_{g}$. It is insightful to compare these results with the CSDs, since AP squirmers with $\beta<3$ have monomodal CSDs, with the width of the distribution depending on the value of $\beta$. Nonetheless, Fig. 10(a) shows that clusters morphology is independent of the hydrodynamic signature when $\beta<3$, but once $\beta=3$ the CSD is bimodal and it turns out that the change in $R_{g}$ coincides with the changes in the CSD. Moreover, dynamic clusters are observed when $\beta<3$ while less dynamic ones are formed when $\beta=3$; therefore, attractive interaction is enhanced when particles are strong pullers.

Similarly, $R_{g} \sim s^{0.64}$ in Fig. 10(b), when the interaction range is short and for small clusters $(s<50)$, while for larger clusters of pullers $(s>50)$ the $R_{g}$ power law is smaller than 0.64. The slower growth of $R_{g}$ depends on the fact that the clusters' structures are mostly controlled by hydrodynamics.
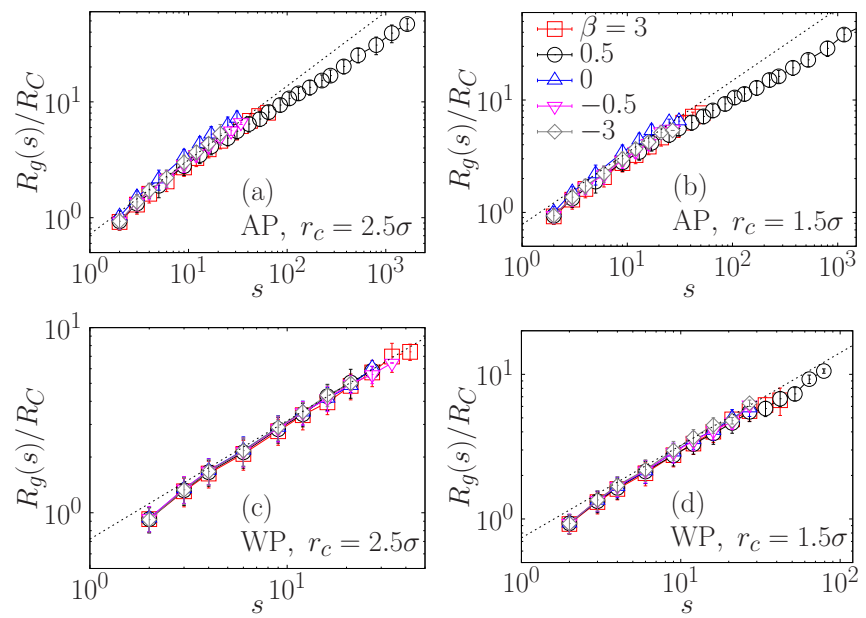

FIG. 11. Radius of gyration normalized by the particle radius as a function of the cluster size for suspensions with $\xi=10$. Top panels are AP squirmers (a) with $r_{c}=2.5 \sigma$ and (b) $r_{c}=1.5 \sigma$, whereas bottom panels are WP squirmers (c) with $r_{c}=2.5 \sigma$ and (d) $r_{c}=$ $1.5 \sigma$. Gray dashed lines in all panels are the function $R_{g}=K s^{0.64}$ and most of the curves fit with this power law.

For WP squirmers, $R_{g}$ shows a richer behavior: Particles first aggregate in trimers and then organize in chainlike structures. As in Fig. 10(c), $R_{g}$ presents a crossover to a different asymptotic regime.

Chains are observed only for WP independently of $\beta$; hence, chain formation is driven by the anisotropic interaction and not by hydrodynamics and is rather insensitive to the range of the potential, as in Fig. 10(d).

When self-propulsion dominates over attraction, $R_{g}=$ $k s^{0.64}$ for small clusters of AP squirmers independently on $\beta$, while for large clusters of pullers $R_{g}$ has a smaller exponent Fig. 11(a). The same happens when the attraction range is $1.5 \sigma$, Fig. 11(b). In Fig. 11(c) we report the radius of gyration for the WP squirmers when $r_{c}=2.5 \sigma$. Given that activity dominates over attraction, particles do not assembly as chains: $R_{g}$ does not present the crossover as in Fig. 10(c), but rather $R_{g} \sim s^{0.64}$ for all $s$. Moreover, the clusters' morphology is the same despite of the active stress $\beta$. However, when the interaction range is reduced to $r_{c}=1.5 \sigma$, as in Fig. 11(d), clusters of pullers are more compact when larger than 20 particles.

\section{E. Local polar and nematic order}

We now analyze the degree of alignment as a function of the cluster size, computing in Fig. 12 the polar order $P(s)$ when $\xi=1$ using Eq. (9). In general, the polar order inside a cluster usually decreases with cluster size as a power law [26].

As reported in Fig. 12(a), increasing $|\beta|$ leads to a faster decay of the local polar order for AP squirmers with $\xi=1$ and $r_{c}=2.5 \sigma$. When decreasing the interaction range, we observe a stronger dependence of the polar order with the hydrodynamic signature: clusters of neutral squirmers [blue triangles in Fig. 12(b)] are practically swimming in the same direction, while clusters of weak pullers [black circles in Fig. 12(b)] show different behavior depending on their size: 

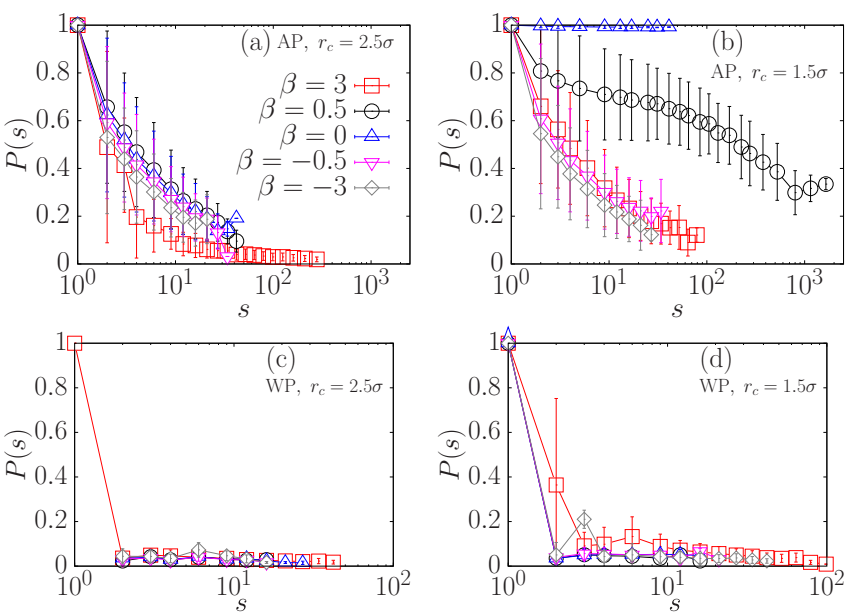

FIG. 12. Polar order as a function of cluster size for $\xi=1$. Top panels: AP squirmers with (a) $r_{c}=2.5 \sigma$ and (b) $r_{c}=1.5 \sigma$, whereas bottom panels are WP squirmers with (c) $r_{c}=2.5 \sigma$ and (d) $r_{c}=$ $1.5 \sigma$.

For small and medium size (up to 100 particles) the polar order decays with size monotonically; the polar order has a crossover for larger clusters, decreasing faster with the cluster size. In general, weak pullers present higher degree of polar order than pushers and strong pullers behave in the same way as the corresponding pushers [with $P(s)$ decaying with the cluster-size monotonically].

However, the results of the local polar order for WP clusters are quite different. Given that squirmers form elongated clusters from the assembly of trimers, the local order rapidly vanishes as the cluster size grows [Fig. 12(c)]. This tendency is less marked for short-range anisotropic interactions and large $\beta$ [strong pullers in Fig. 12(d) (red squares)].

In Fig. 13 we report the polar order as a function of the cluster size for both AP and WP squirmers when selfpropulsion dominates over attraction $(\xi=10)$.

For AP squirmers, $P(s)$ decays faster for pushers than for pullers, independently on the interaction range [Figs. 13(a)
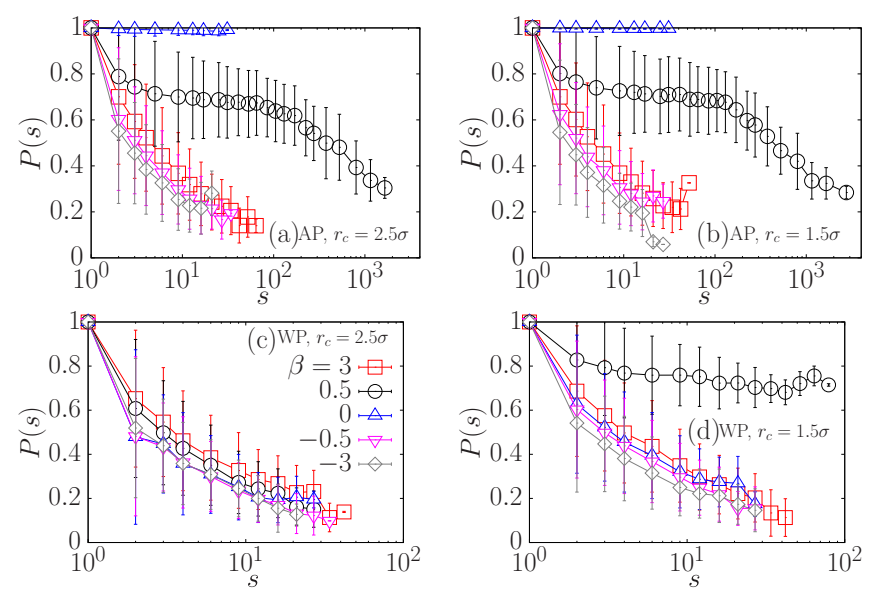

FIG. 13. Polar order as a function of cluster size for $\xi=10$. Top panels: AP squirmers with (a) $r_{c}=2.5 \sigma$ and (b) $r_{c}=1.5 \sigma$, whereas bottom panels are WP squirmers with (c) $r_{c}=2.5 \sigma$ and (d) $r_{c}=$ $1.5 \sigma$.
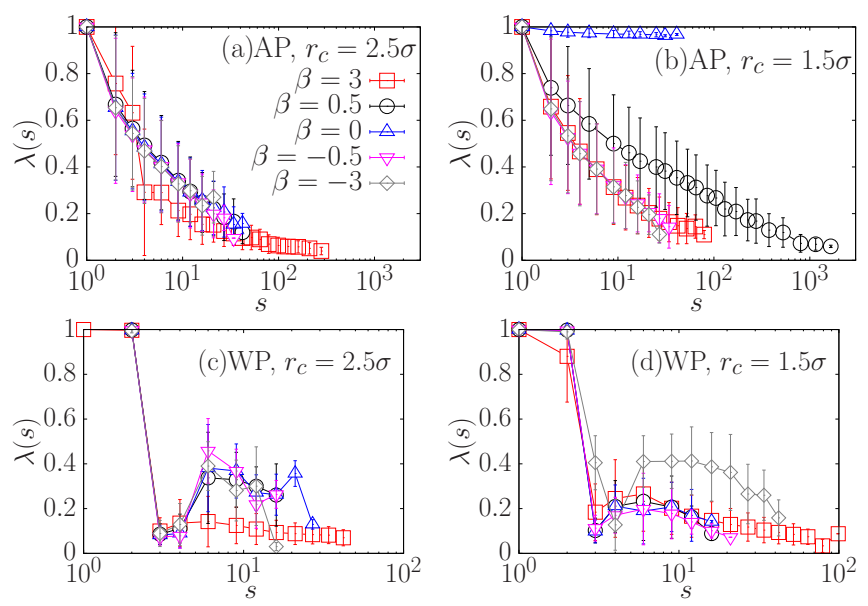

FIG. 14. Nematic order as a function of cluster size when $\xi=1$. Top panels represent results for AP squirmers: with (a) $r_{c}=2.5 \sigma$ and (b) $r_{c}=1.5 \sigma$, whereas bottom panels are WP squirmers with (c) $r_{c}=2.5 \sigma$ and (d) $r_{c}=1.5 \sigma$.

and 13(b)]. The degree of polar order is more sensitive to $\beta$ for pullers than for pushers; weak puller's clusters are more aligned than clusters of stronger pullers, while neutral squirmer's clusters are the most aligned ones for all cluster sizes. Figures 13(c) and 13(d) report the polar order of clusters of WP squirmers, with a monotonous decay of the polar order with cluster size, weakly dependent on $\beta$ and on the interaction range (except for $\beta=0.5$ ).

In Fig. 14 we report results for the nematic order $\lambda(s)$. The local nematic order for AP squirmers with $\xi=1$ decays with the cluster size $s$ independently on $\beta$ for $\beta<3$ while presents an even faster decay when $\beta=3$ [see Fig. 14(a)]. When $r_{c}=1.5 \sigma, \lambda(s)$ decays with $s$ in the same way as $P(s)$ does for all $\beta$. Neutral squirmers are completely polarized [as also shown in the nematic order, blue triangles in Fig. 14(b)]. The high polar order observed in clusters of puller and neutral squirmers is also detected by the nematic order.

The behavior of the local nematic order for WP clusters with $\xi=1$ is quite striking. The reason why $\lambda(1)=\lambda(2)=1$ is that when the interaction range is $2.5 \sigma$, once two particles collide they are kept together by the attractive patch. Particles in trimers and tetramers point toward the center of the cluster and are kept together by the attractive patch, reason why they have a small nematic order [all curves in Fig. 14(c)]. For larger clusters, active stresses become more important: Hydrodynamics attract trimers so that particles' attractive patches reorient in the clusters forming aligned chains. This does not happen for strong pullers, where the nematic order remains low and constant [red squares in Fig. 14(c)], due to the fact that trimers form disordered chains (made of trimers formed by hydrodynamics). When the interaction range is $1.5 \sigma$, the local nematic order behaves in the same way as for $2.5 \sigma$, but in this case oriented chains are observed only for strong pushers, whereas the rest of squirmers form lessoriented chains with a value of $\lambda(s)$ in between.

In Figs. 15(a) and 15(b) we report the local nematic order $\lambda(s)$ for $\xi=10$ and AP squirmers. In clusters made of neutral squirmers (blue triangles), particles swim in the same direction independently on the interaction range: $\lambda(s) \approx 1$ for all 

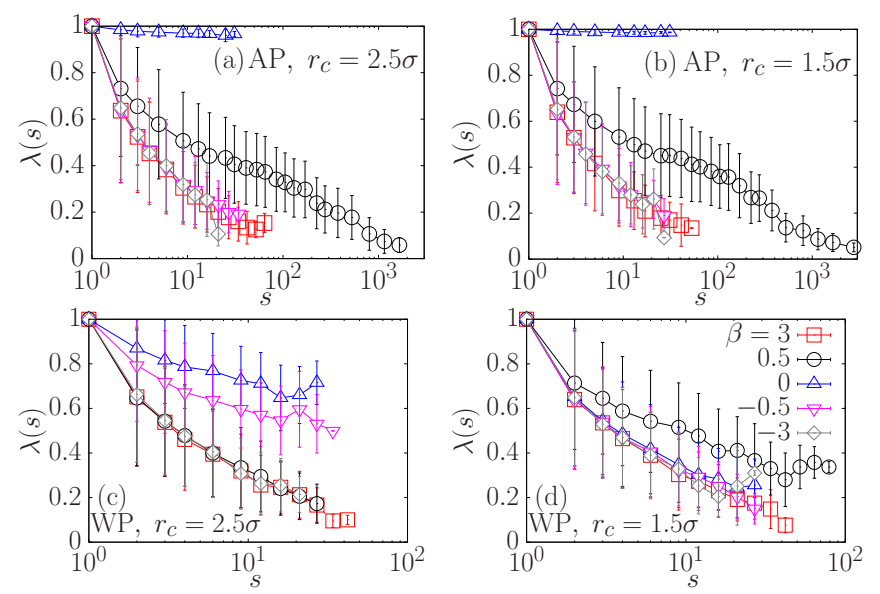

FIG. 15. Nematic order as a function of cluster size for suspensions with $\xi=10$. (a) AP squirmers with $r_{c}=2.5 \sigma$. (b) AP squirmers with $r_{c}=1.5 \sigma$. (c) WP squirmers with $r_{c}=2.5 \sigma$. (d) WP squirmers $r_{c}=1.5 \sigma$.

cluster sizes. The nematic order for all other squirmers decays in the same way as the polar order. To conclude, in the case of clusters of AP squirmers, alignment is not affected by the interaction range.

WP clusters with $\xi=10$ and long-range interactions develop a high nematic order for neutral and weak pushers ( $\beta=0$ and -0.5$)$ as shown in Fig. 15(c). This nematic order is not due to a global polar order (being very low) but to the competition between the anisotropic potential and the hydrodynamic signature: Even though activity dominates over attraction, the interaction range is long enough to allow for the development of a nematic order in the system. Undoubtedly, the interaction range is important to develop clusters with nematic order, since $\lambda(s)$ for WP squirmers with short range has a different behavior, where the nematic order only shows a nonzero value due to the polar order shown before.

\section{F. Global polar and nematic order}

To study the orientational order of the system, we compute as in Ref. [10] the global polar order parameter and the nematic order tensor Eq. (10) [55] at steady state at long times. In Fig. 16(a) we represent the global polar order $P_{\infty}$ for AP squirmers in steady state for $\xi=1$ and $\xi=10$ and both interaction ranges.

When $\xi=10$, activity dominates and the polar order parameter has a nonzero value for weak pullers $(0 \geqslant \beta \geqslant 1)$, similarly to the 3D case without attractive interactions [10]. When $\xi=1$, the range of interaction affects to the formation of aligned states: For $2.5 \sigma$ the system is completely isotropic despite the value of $\beta$ [red circles in Fig. 16(a)], whereas for $r_{c}=1.5 \sigma$ the system still has polar order for weak pullers (black circles), as with $\xi=10$. Figure 16(b) shows that the nematic order $\lambda_{\infty}$ follows the polar order: For AP squirmers there is not an additional orientation besides the polar order.

In Fig. 16(c) we represent the global polar order $P_{\infty}$ for WP squirmers in steady state for $\xi=1$ and $\xi=10$ and both interaction ranges. For strongly anisotropic interactions $(\xi=$ 1) the suspension does not develop any significant degree of polar ordering. For weakly interacting WP squirmers $(\xi=10)$
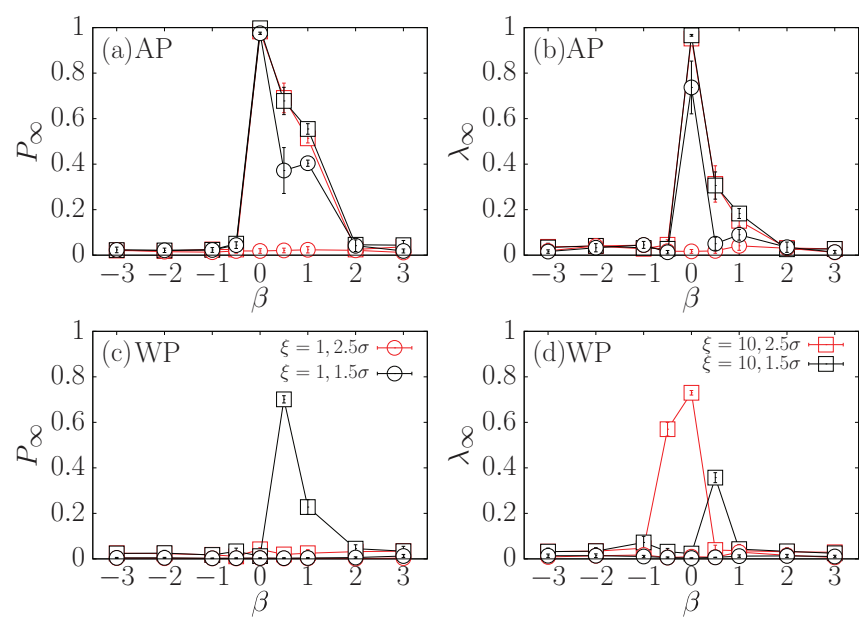

FIG. 16. Global polar $P_{\infty}$ and nematic $\lambda_{\infty}$ order parameters. Top panels represent results for AP squirmers: (a) polar order parameter $P_{\infty}$ and (b) nematic order parameter $\lambda_{\infty}$. Bottom panels represent results for WP squirmers: (c) polar order parameter $P_{\infty}$ and (d) nematic order $\lambda_{\infty}$.

and $r_{c}=1.5 \sigma$, the polar order emerges in the same range of $\beta^{\prime}$ s (black squares) as the AP squirmers. In Fig. 16(d) we report the nematic order parameter $\lambda_{\infty}$. When $\xi=1 \mathrm{WP}$ squirmers do not develop any nematic order (red and black circles): Either polar or nematic order are absent for WP squirmers with strongly anisotropic interactions. A nonzero nematic order is observed for $\xi=10$, when $r_{c}=2.5 \sigma$ and $r_{c}=1.5 \sigma:$ In the latter, the nematic order is due to the polar order (black squares), while in the former midrange interactions a new orientational order (red squares) is created. Interestingly, the nematic order parameter for WP squirmers with weak interactions shows a nonzero nematic order when the polar order parameter is zero.

\section{DISCUSSION AND CONCLUSIONS}

In this paper, we report a numerical study of the selfassembly of Janus squirmers in a quasi-two-dimensional semidilute suspension.

Even though we are aware of the fact that experimental catalytic active colloids might present other types of interactions, like phoretic ones, the nature of such interactions is going to depend on the particular system under study [33]. As in the case of active dipolar Janus particles [36] where electrophoresis is effectively modelled by the interaction between two imbalanced charges on each particle, in our work we suggest that amphiphilic active Janus colloids [35] can be modelled by means of an effective anisotropic interaction. For this $2 \mathrm{D}$ system, we study the effect of the hydrodynamic interactions in the self-assembly of such particles, hopefully shedding some light on experiments with active Janus colloids where the fluid flow field can be measured [39] or in experiments where the self-propulsion direction can be tuned [38].

In a previous work, we have studied aggregation in a semidilute quasi-two-dimensional suspension of isotropically attractive squirmers [26], demonstrating that both clusters' morphology and alignment within clusters depended on particles' interaction strength and hydrodynamic signature. 
In the current work, by changing the symmetry of the interparticle interaction (from isotropic to anisotropic) we have observed that the interaction anisotropy, modulated by its range and strength, compete with the active stress to reorient the particles, therefore giving rise to a rich aggregation phenomenology.

The study of two different interaction ranges was originated from previous results [22,23,25,26,58], where $r_{c}=$ $2.5 \sigma$ has been used for isotropic potentials, while in Refs. $[37,59,60]$ an interaction range of $r_{c}=1.5 \sigma$ is used for anisotropic potentials. Both cutoff values are typical in the context of attractive Brownian colloids either passive or active.

As shown in all studied cases, the amount of activity versus attraction has a strong effect on the aggregation dynamics, affecting not only the characteristic cluster size $\langle s\rangle$ but also the timescale at which the steady state (if any) is reached.

When the attractive interaction dominates over propulsion, e.g., at $\xi=0.1$, AP squirmers coarsen and the velocity of coarsening will depend on the hydrodynamic stresses for both interaction ranges, similarly to the isotropic case with lower interaction strength $(\xi=0.7)$ [26], where the system coarsens for pullers and weak pullers and forms clusters for strong pushers. As opposed to AP, WP squirmers develop more striking effects; for long-range interactions squirmers coarsen very slowly as far as our calculation time is concerned. While for short range, WP squirmers have reached a clustering steady state, where clusters are self-assembly from small chains or trimers. WP cases at $\xi=0.1$ are explained deeply in Appendix D.

AP squirmers display a behavior closer to that of squirmers interacting with an isotropic potential when interaction competes with activity and when activity dominates over attraction [26]. The competition between interaction and activity always in terms of $\xi$, for example, the fluctuation of the mean cluster size for weak pullers observed in Fig. 4(b) and Figs. 5(a) and 5(b) or the power-law behavior of the CSD for weak pullers in Fig. 8(b) and Figs. 9(a) and 9(b). In contrast, WP squirmers have a stronger tendency to develop novel collective behavior, from polydisperse suspension of monomers, dimers, trimers, and even chains of tens of particles. Our interpretation here is that, since two AP squirmers interact when their attractive patches are within their range, this effect is reduced due to their propulsion in opposite directions. Thus the contribution to the torque is mostly given by hydrodynamic stresses rather than by the anisotropic interactions, as in the isotropic case.

In order to perform a statistical study of the clusters, we focus on $\xi=1$ and $\xi=10$ when the system is in steady state. We first compute the CSD. On the one side, CSD for AP squirmers can be described by a power law with an exponential tail. When $\xi=1$, CSDs are characterized by a cutoff-algebraic shape except for strong pullers, which develop a bimodal distribution not present for short interaction range $(1.5 \sigma)$. In this case, the CSD's width follows a behavior reminding that of isotropic squirmers with $\xi>1$, i.e., wider CSDs for weak pullers. When $\xi=10$, the effect of the interaction range on the CSDs is not observed for any value of $\beta$. On the other side, the CSDs for WP squirmers has a peak for trimers when $\xi=1$. The shape of the CSDs is approximately the same independently on the attraction range.
When $\xi=10$, even though the CSD is described by a power law with an exponential tail, when $r_{c}=2.5 \sigma$ the larger the value of $\beta$ the wider the distribution; whereas when $r_{c}=1.5 \sigma$ the shape of the CSD is mainly dictated by hydrodynamics, with weak pullers showing a wider distribution.

Next we compute the radius of gyration as a function of the cluster size. When $\xi=1$ and $r_{c}=2.5 \sigma$, the morphology of AP squirmers follows the same behavior as the one detected in the isotropic case [26], with more compact clusters for strong pullers; differently from the case when $r_{c}=1.5 \sigma$, where clusters are more compact for weak pullers. When $\xi=10$ the clusters' $R_{g}$ does not change, independently on the interaction range or the hydrodynamic signature. When $\xi=1$, the $R_{g}$ for WP squirmers varies with the clusters sizes since, for both interaction ranges, trimers and tetramers gives rise to the formation of chains, which lead to different exponents. In contrast, when $\xi=10$ and $r_{c}=2.5 \sigma$, the $R_{g}$ for WP squirmers is the same despite $\beta$; however, when $r_{c}=1.5 \sigma$ pullers form more compact clusters.

It is worth mentioning that these chains resemble the chains observed with amphiphilic active Brownian particles [37], but clearly their nature is different since WP squirmers form chains due to their stresslet, while the Brownian case depends on the activity and the size of the hydrophobic patch (see Appendix B). Moreover, it seems that hydrodynamic interactions cancel the formation of chains for AP squirmers, since we have observed chains just for WP squirmers.

To establish a potential alignment within clusters, we compute both polar and nematic order. When $\xi=1$, the polar order of the clusters for AP squirmers strongly depends on $r_{c}$ : when $r_{c}=2.5 \sigma, P(s)$ quickly decays with the cluster size (quicker for pushers than for pullers); whereas when $r_{c}=1.5 \sigma, P(s)$ corresponds to high polar order for large clusters with $\beta=0$ and weak pullers. The same features can be observed when $\xi=10$, independently on $r_{c} . P(s)$ for WP squirmers vanishes for all cluster sizes, independently of $\beta$ and $r_{c}$ when $\xi=1$, since the formation of trimers and chains assemble the particles head to head, thus it avoids the polar order. However, when $\xi=10$, the polar order decays with $s$ : For $r_{c}=1.5 \sigma$ weak pullers form clusters with high polar order, while for $r_{c}=2.5 \sigma P(s)$ decays faster for pushers than for pullers. In the latter case, the WP squirmers show another type of alignment, with particles with a high nematic order in the range of $\beta=0$ and weak pushers.

During aggregation, we have identified seven different cases depending on whether the attractive patch is oriented against the propulsion direction (AP squirmers) or directed toward it (WP squirmers): three gas states, three clustering cases, and one coarsening. On the one side, AP squirmers coarsen isotropically if the interaction is strong enough $\xi \approx 0.1$. When the interaction strength competes with selfpropulsion $(\xi=1)$, dynamic clusters emerge whose mean size depends on hydrodynamic stresses. When activity dominates ( $\xi=10)$, particles form a gas and depending on the value of $\beta$ this gas can be isotropically oriented or polarly oriented. On the other side, WP squirmers form chains of particles if the interaction is high enough $(\xi \approx 0.1)$, or a suspension of trimers and tetramers if the interaction competes with the active propulsion $(\xi=1)$. Whenever activity dominates $(\xi=10)$, particles form a gas and depending on the value 
of $\beta$ this gas can be isotropic, polar, or even nematically oriented.

Therefore, on one hand anisotropy drives the formation of structures of trimers and tetramers for WP squirmers; on the other hand, anisotropy modulates the sensitivity of the hydrodynamic signature: While WP squirmers are more sensitive to $r_{c}$ when $\xi=10$, AP squirmers are more sensitive to the interaction range $\left(r_{c}\right)$ when $\xi=1$. To conclude, the rich morphology of the detected squirmers' aggregates is the result of the anisotropic interactions, characterized by the angular attractive potential and its interaction range, in competition with the active stress, that can be pointing toward or against the attractive patch.

\section{ACKNOWLEDGMENTS}

We thank S. A. Mallory and A. Cacciuto for helpful discussions. This work was possible thanks to the access to MareNostrum Supercomputer at Barcelona Supercomputing Center (BSC). This article is based on work from COST Action MP1305, supported by COST (European Cooperation in Science and Technology). This work was supported by Grant No. FIS2016-78847-P of the MINECO and the UCM/ Santander No. PR26/16-10B-2. I.P. acknowledges MINECO and DURSI for financial support under Projects No. FIS201567837- P and No. 2017 SGR-884, respectively. F.A. acknowledges funding from MINECO Juan de la Cierva-formación program: FJCI-2017-33580.

\section{APPENDIX A: ATTRACTIVE PART OF THE POTENTIAL}

Figure 17 compares the radial part of the total short-range potential given by Eq. (4) (red curves) and the classical LJ potential Eq. (3) and cut-off distance of $2.5 \sigma$ (blue curves), $\sigma$ is the particles' diameter. Both curves in Fig. 17 are the sum of

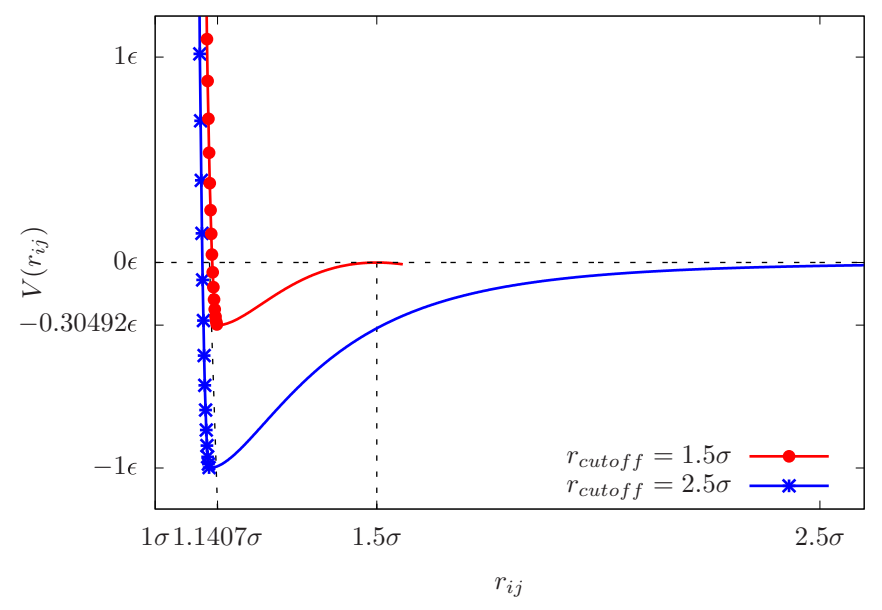

FIG. 17. Graphic representation of the total potential $V\left(r_{i j}\right)$ with $\phi=1$, using the Lennard-Jones potential with a smooth transition function given in Eq. (4) as the attractive part of $V\left(r_{i j}\right)$ (red curve), the repulsive soft sphere potential at short separation distance with $h_{0}=\left(2.2^{1 / 6}-1\right) \sigma$ (red points) is using with this cut-off potential. The blue curve is using Eq. (3), with the repulsive soft sphere potential at short separation distance with $h_{0}=\left(2^{1 / 6}-1\right) \sigma$ (blue asterisks).

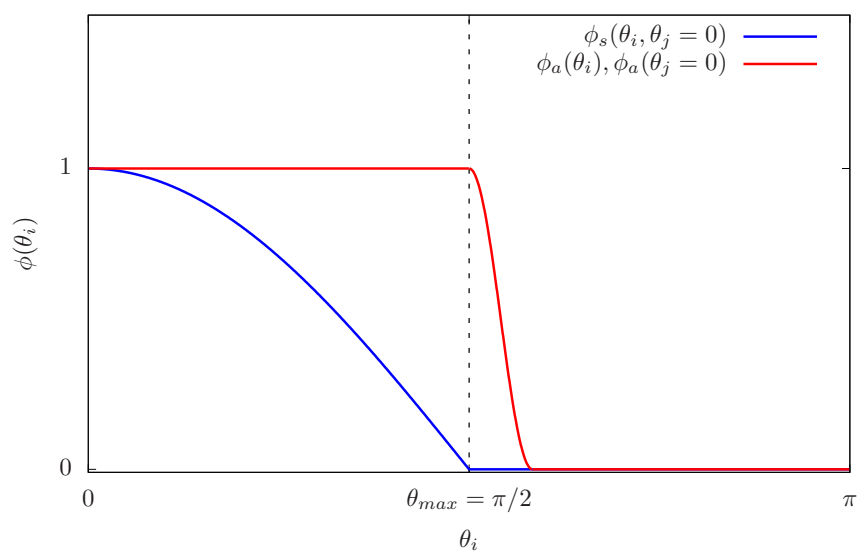

FIG. 18. Graphical representation of the angular dependence of two different Janus potentials. In blue the symmetric potential used in our simulations given by Eq. (5), while in red the asymetric potential used in Refs. [37,59] and depicted in Eq. (B1). In order to compare both angular functions, we set $\theta_{j}=0$ in both cases and $\theta_{\max }=\pi / 2$ which corresponds to the symmetric case.

the repulsive soft-sphere potential [Eq. (2)] and the respective attractive potential.

\section{APPENDIX B: DIFFERENT TYPES OF JANUS INTERACTIONS}

There are different models to simulate the amphiphilic behavior of Janus colloids, and in this Appendix we have compared two different models for the angular potential $\phi$ in Eq. (1). The angular potential used in this manuscript [Eq. (5)] has been used to simulate spherical particles with one hydrophobic hemisphere and charged on the other [45] where both hemispheres have the same size, thus let us call it symmetric potential $\phi_{s}\left(\theta_{i}, \theta_{j}\right)$.

On the other hand, in Ref. [59], they developed an asymmetric potential where the size of the hydrophobic region is tuned in order to study 3D Janus colloid suspensions in equilibrium and more recently, some of us have used this potential to study the self-assembly of active amphiphilic Janus particles with attractive patches of different sizes [37]. Analogously to the symmetric potential above, the the orientation depending term is given by

$$
\begin{aligned}
\phi_{a}\left(\theta_{i}\right) & \theta_{i} \leqslant \theta_{\max } \\
= & \left\{\begin{array}{lc}
1 & \theta_{\max } \leqslant \theta_{i} \leqslant \theta_{\max }+\theta_{\text {tail }} \\
\cos ^{2}\left(\frac{\pi\left(\theta_{i}-\theta_{\max }\right)}{2 \theta_{\text {tail }}}\right) & \text { otherwise. } \\
0 &
\end{array}\right.
\end{aligned}
$$

The orientational part of the potential $\phi_{a}$ is a a smooth step function that modulates the angular dependence of the potential, the smoothness is modulated by the parameter $\theta_{\text {tail }}$, which it is set to $\theta_{\text {tail }}=0.2618$ in Ref. [59] for 3D Janus colloids while in Ref. [37] was tuned in $\theta_{\text {tail }}=0.436$ to generate a sufficiently smooth potential at the Janus interface, and $\theta_{\max }$ tunes the size of the hydrophobic region for a patch coverage of $50 \%$ then $\theta_{\max }=\pi / 2$.

By the analysis of the curves shown in Fig. 18, it is clear that interactions among the Janus particles will depend on the 

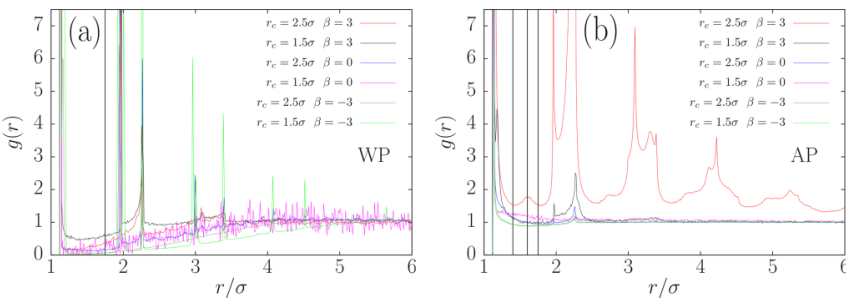

FIG. 19. Radial distribution functions $g(r)$ with $\xi=1$ for aligned with the patch interaction (left) and aligned against the patch interaction (right).

angular function we choose, despite the fact that $\theta_{\max }=\pi / 2$ in the asymmetric potential.

In terms of the interaction strength, it is clear that $\phi_{s}$ will give us a lower attraction than $\phi_{a}$ for any direction of $\theta_{i}$. An important remark is that $\phi_{s}=0$ when two particles are parallel while $\phi_{a} \neq 0$ in the same configuration, and therefore $\phi_{a}$ enhances the emergence of chainlike aggregates [37].

To understand the relevance of the orientational part in the potential and the hydrodynamic interactions, we are working in a model of amphiphilic squirmers using $\phi_{a}$ in order to compare with the "dry" case of Ref. [37], this work is in progress.

\section{APPENDIX C: IDENTIFYING CLUSTERS IN THE SUSPENSION}

In order to determine the criterion to characterize whether a particle belongs or not to a cluster, we computed first the radial distribution function of our swimmer suspensions.

We observe a local peak at $r=1.6 \sigma$ for the $g(r)$ corresponding to $\beta=3$ with $r_{c}=2.5 \sigma$ with AP interaction. We took $r_{\mathrm{cl}}=1.75 \sigma$ for all cases, although for this case it is evident the first minimum cannot be located there. We analyze how the mean cluster size and the cluster size distribution vary for this troublesome case with the choice of $r_{\mathrm{cl}}$, comparing $r_{\mathrm{cl}}=1.75 \sigma$ to a cutoff in the peak $r_{\mathrm{cl}}=1.6 \sigma$ and a cutoff lying in the first valley $r_{\mathrm{cl}}=1.4 \sigma$ [Fig. 19(a) and 19(b)].

We observe that as we reduce $r_{\mathrm{cl}}$, the mean cluster size is reduced, being the difference between $r_{\mathrm{cl}}=1.75 \sigma$ and $r_{\mathrm{cl}}=$ $1.4 \sigma$ of 0.9 units $(\approx 10 \%$ ) [Fig. 20(a)]. As for the cluster size distribution, we find that all three of them are very similar. Even if the frequencies for the largest observed clusters are reduced with $r_{\mathrm{cl}}$, all distributions are cut at the same bin (clusters sized between 240 and 318 particles), with a frequency $7.7 \times 10^{-6}$ for $s=279$ and $r_{\mathrm{cl}}=1.75 \sigma$, and $4.6 \times 10^{-6}$ for the same size with $r_{\mathrm{cl}}=1.4 \sigma$. The monomer frequency goes
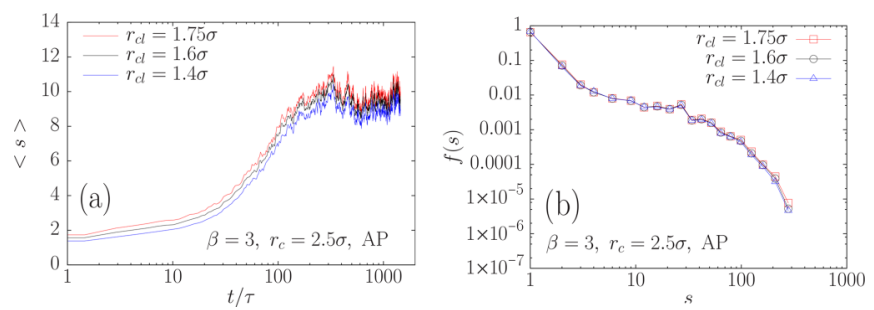

FIG. 20. Left: Time evolution of the mean cluster size. Right: Cluster size distribution.

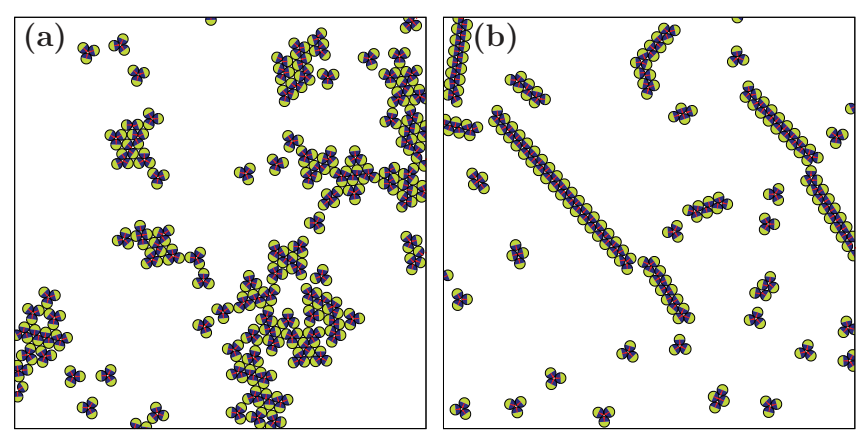

FIG. 21. Simulation zoomed snapshots of WP squirmers with $\xi=0.1$ and $r_{c}=2.5 \sigma$ snapshots are taken at the last time step of the simulations. The attractive hemisphere is represented in blue, while pure repulsive is in green and the fixed orientation vector is shown in red. (a) $\beta=3$ and (b) $\beta=-3$.

from 0.680 for $r_{\mathrm{cl}}=1.4 \sigma$ to 0.656 for $r_{\mathrm{cl}}=1.75 \sigma$, and all three curves have a very similar shape, sharing even local maxima and minima along the whole profile [Fig. 20(b)].

\section{APPENDIX D: CLUSTERING ANALYSIS FOR WP JANUS WITH $\xi=0.1$}

Janus squirmers with interaction strength $\xi=0.1$ develop very interesting dynamics; on one hand, for strong pullers with $\beta=3$, particles form mainly trimers and tetramers that eventually aggregate due to hydrodynamic interactions [Fig. 21(a) and Fig. 22(a)]. The other case is the chains system, where particles self-assemble in chains of different sizes, and such chains are formed by two lines of squirmers with the attractive patch pointing toward the other line of squirmers that form the chain [Fig. 21(b) and Fig. 22(b)]. The chain length depends on the value of $\beta$. This chain system has been observed for pushers and weak pullers as well.

When interaction range is $r_{c}=2.5 \sigma$, the clusters, either trimers or chains, keep growing slowly; as far as our calculation capacity could reach, we have observed coarsening for all $\beta$ in contrast with WP squirmers with an interaction range of $r_{c}=1.5 \sigma$. In this case WP squirmer suspensions reach a

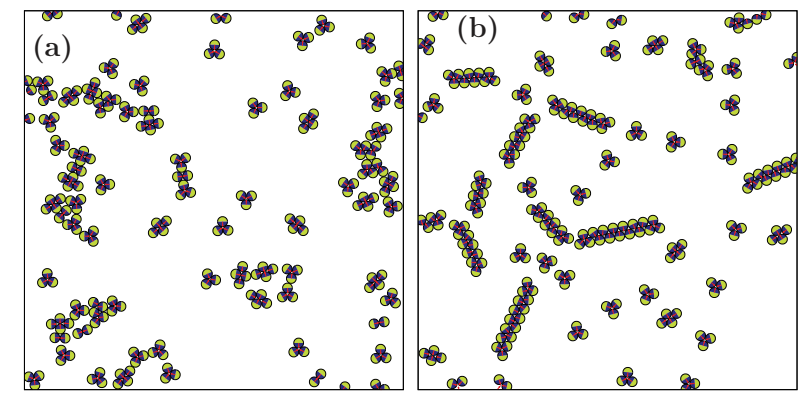

FIG. 22. Simulation zoomed snapshots of WP squirmers with $\xi=0.1$ and $r_{c}=1.5 \sigma$ snapshots are taken once the simulations reach a steady state given by the mean cluster size. The attractive hemisphere is represented in blue, while pure repulsive in green and the fixed orientation vector is shown in red. (a) $\beta=3$ and (b) $\beta=-3$. 

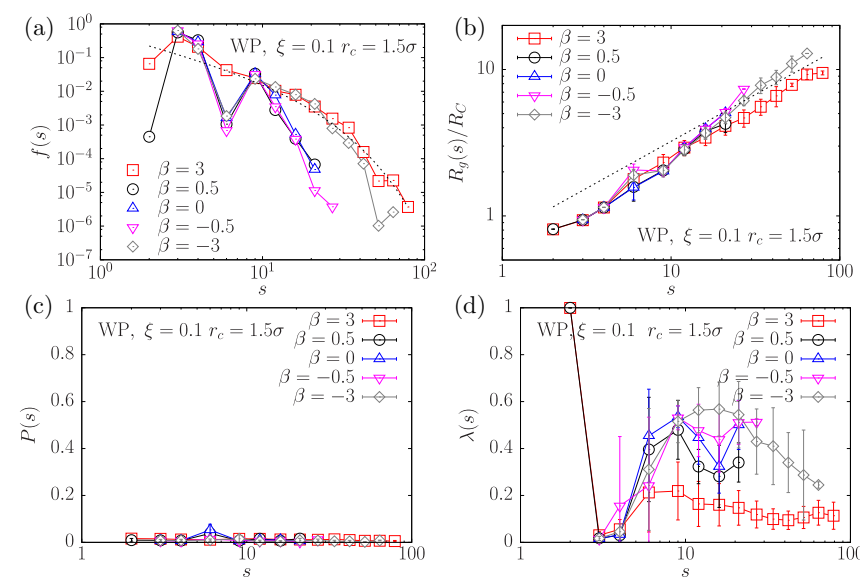

FIG. 23. Cluster analysis of WP squirmers with $\xi=0.1$ and $r_{c}=1.5 \sigma$. (a) Cluster size distribution. (b) Radius of gyration as a function of cluster size. (c) Local polar order and (d) local nematic order parameters. Pullers curves are red and black, while pushers are pink and gray and $\beta=0$ in blue. Note the different scale on the $y$ axis.

clustering state for all hydrodynamic signature $\beta$. We have found two general types of clustering.

We have characterized the clusters observed for these WP squirmers. In [Fig. 23(a)] we show the cluster size distribution $f(s)$. In general, $f(s)$ has two main peaks, one for trimers and another one in the bin of cluster-size around 10 particles for pushers and weak pullers, while strong pullers (red squares) have just one peak for trimers. Moreover, none of these distributions show monomers and just pullers show dimers in their distributions. Another interesting feature of the cluster size distributions are the fact that both strong pushers and pullers have bigger clusters than the rest of squirmers (gray diamonds and red squares respectively) and both distributions follow the analytical shape of Eq. (11) (dotted line) for large clusters.

Even though both cluster size distributions are similar for strong pushers and pullers, the morphology of such clusters are not the same. As we can observe in Fig. 23(b), where despite the fact that the radius of gyration for small clusters $(s \leqslant 10)$ follows the same behavior for all $\beta$, the large clusters for strong pullers (red squares) are more compact (small slope) than clusters of the rest of squirmers; in fact, clusters of pushers and weak pullers are chains, and thus we can observe a greater slope for large clusters (gray diamonds and pink triangles).

We have also calculated both polar and nematic order parameters at each cluster, and we have observed that clusters have no polar order at any value of $\beta$ [Fig. 23(c)], but an alignment, given by a nonzero nematic order, is observed [Fig. 23(d)]. For pullers, all dimers are aligned in a head-to-head configuration and then the nematic order is 1 ; particles in trimers and tetramers in all cases are pointing out to the center of the clusters and therefore the nematic order is zero. But, in the case of pushers to weak pullers, all clusters have a large nonzero nematic order, since particles are forming chains of two lines of particles aligned to the center of the chain giving rise to a nematic order. In contrast, for strong pullers (red squares) large clusters are formed by trimers gather together by hydrodynamic interactions, and thus particles are pointing in different directions and nematic order parameter is reduced. In fact, the magnitude of nematic order parameter for large clusters is given by the $\beta$ value. The lower the $\beta$ the greater the nematic order.
[1] C. Dombrowski, L. Cisneros, S. Chatkaew, R. E. Goldstein, and J. O. Kessler, Phys. Rev. Lett. 93, 098103 (2004).

[2] L. H. Cisneros, R. Cortez, C. Dombrowski, R. E. Goldstein, and J. O. Kessler, Exp. Fluids 43, 737 (2007).

[3] T. Ishikawa, M. Simmonds, and T. Pedley, J. Fluid Mech. 568, 119 (2006).

[4] T. Ishikawa and T. J. Pedley, J. Fluid Mech. 588, 437 (2007).

[5] T. Ishikawa, J. T. Locsei, and T. J. Pedley, J. Fluid Mech. 615, 401 (2008).

[6] T. Ishikawa and T. J. Pedley, Phys. Rev. Lett. 100, 088103 (2008).

[7] J. J. Molina, Y. Nakayama, and R. Yamamoto, Soft Matter 9, 4923 (2013).

[8] N. Oyama, J. J. Molina, and R. Yamamoto, Phys. Rev. E 93, 043114 (2016).

[9] N. Oyama, J. J. Molina, and R. Yamamoto, J. Phys. Soc. Jpn. 86, 101008 (2017).

[10] F. Alarcón and I. Pagonabarraga, J. Mol. Liq. 185, 56 (2013).

[11] I. Pagonabarraga and I. Llopis, Soft Matter 9, 7174 (2013).

[12] A. A. Evans, T. Ishikawa, T. Yamaguchi, and E. Lauga, Phys. Fluids 23, 111702 (2011).

[13] B. Delmotte, E. E. Keaveny, F. Plouraboué, and E. Climent, J. Comput. Phys. 302, 524 (2015).
[14] J. Palacci, S. Sacanna, A. P. Steinberg, D. J. Pine, and P. M. Chaikin, Science 339, 936 (2013).

[15] J. Schwarz-Linek, C. Valeriani, A. Cacciuto, M. E. Cates, D. Marenduzzo, A. N. Morozov, and W. C. K. Poon, Proc. Natl. Acad. Sci. USA 109, 4052 (2012).

[16] A. P. Petroff, X.-L. Wu, and A. Libchaber, Phys. Rev. Lett. 114, 158102 (2015).

[17] I. Theurkauff, C. Cottin-Bizonne, J. Palacci, C. Ybert, and L. Bocquet, Phys. Rev. Lett. 108, 268303 (2012).

[18] S. Thutupalli, R. Seemann, and S. Herminghaus, New J. Phys. 13, 073021 (2011).

[19] A. Zöttl and H. Stark, Phys. Rev. Lett. 112, 118101 (2014).

[20] J. Blaschke, M. Maurer, K. Menon, A. Zöttl, and H. Stark, Soft Matter 12, 9821 (2016).

[21] R. Matas-Navarro, R. Golestanian, T. B. Liverpool, and S. M. Fielding, Phys. Rev. E 90, 032304 (2014).

[22] G. S. Redner, A. Baskaran, and M. F. Hagan, Phys. Rev. E 88, 012305 (2013).

[23] B. M. Mognetti, A. Šarić, S. Angioletti-Uberti, A. Cacciuto, C. Valeriani, and D. Frenkel, Phys. Rev. Lett. 111, 245702 (2013).

[24] C. Tung, J. Harder, C. Valeriani, and A. Cacciuto, Soft Matter 12, 555 (2016). 
[25] R. M. Navarro and S. M. Fielding, Soft Matter 11, 7525 (2015).

[26] F. Alarcón, C. Valeriani, and I. Pagonabarraga, Soft Matter 13, 814 (2017).

[27] S. Saha, R. Golestanian, and S. Ramaswamy, Phys. Rev. E 89, 062316 (2014).

[28] O. Pohl and H. Stark, Phys. Rev. Lett. 112, 238303 (2014).

[29] M. Meyer, L. Schimansky-Geier, and P. Romanczuk, Phys. Rev. E 89, 022711 (2014).

[30] B. Liebchen, D. Marenduzzo, I. Pagonabarraga, and M. E. Cates, Phys. Rev. Lett. 115, 258301 (2015).

[31] B. Liebchen, M. E. Cates, and D. Marenduzzo, Soft Matter 12, 7259 (2016).

[32] M. R. Nejad and A. Najafi, Soft Matter 15, 3248 (2019).

[33] B. Liebchen and H. Löwen, J. Chem. Phys. 150, 061102 (2019).

[34] M. Manjare, Y. Ting Wu, B. Yang, and Y.-P. Zhao, Appl. Phys. Lett. 104, 054102 (2014).

[35] W. Gao, A. Pei, X. Feng, C. Hennessy, and J. Wang, J. Am. Chem. Soc. 135, 998 (2013).

[36] J. Yan, M. Han, J. Zhang, C. Xu, E. Luijten, and S. Granick, Nat. Mater. 15, 1095 (2016).

[37] S. Mallory, F. Alarcon, A. Cacciuto, and C. Valeriani, New J. Phys. 19, 125014 (2017).

[38] A. Brown and W. Poon, Soft Matter 10, 4016 (2014).

[39] A. I. Campbell, S. J. Ebbens, P. Illien, and R. Golestanian, arXiv: 1802.04600 .

[40] M. J. Lighthill, Commun. Pure Appl. Math. 5, 109 (1952).

[41] J. R. Blake, J. Fluid Mech. 46, 199 (1971).

[42] T. Pedley, IMA J. Appl. Math. 81, 488 (2016).

[43] M. P. Allen and D. J. Tildesley, Computer Simulation of Liquids (Oxford University Press, Oxford, England, 2017).
[44] S. D. Stoddard and J. Ford, Phys. Rev. A 8, 1504 (1973).

[45] L. Hong, A. Cacciuto, E. Luijten, and S. Granick, Langmuir 24, 621 (2008),

[46] J. L. Anderson and D. C. Prieve, Langmuir 7, 403 (1991).

[47] H. A. Stone and A. D. T. Samuel, Phys. Rev. Lett. 77, 4102 (1996).

[48] M. Cates, J.-C. Desplat, P. Stansell, A. Wagner, K. Stratford, R. Adhikari, and I. Pagonabarraga, Philos. Trans. R. Soc., A 363, 1917 (2005).

[49] S. Succi, The Lattice Boltzmann Equation for Fluid Dynamics and Beyond (Clarendon Press, New York, 2001).

[50] K. Stratford and I. Pagonabarraga, Computers \& Mathematics with Applications 55, 1585 (2008).

[51] N.-Q. Nguyen and A. J. C. Ladd, Phys. Rev. E 66, 046708 (2002).

[52] I. Llopis and I. Pagonabarraga, J. Non-Newtonian Fluid Mech. 165, 946 (2010), Proceedings of the 5th international workshop on non-equilibrium thermodynamics \{IWNET\} 2009.

[53] R. Matas Navarro and I. Pagonabarraga, Eur. Phys. J. E 33, 27 (2010).

[54] C. Valeriani, E. Sanz, P. N. Pusey, W. C. Poon, M. E. Cates, and E. Zaccarelli, Soft Matter 8, 4960 (2012).

[55] D. G. Barci and D. A. Stariolo, Phys. Rev. B 79, 075437 (2009).

[56] R. Eppenga and D. Frenkel, Mol. Phys. 52, 1303 (1984).

[57] See Supplemental Material at http://link.aps.org/supplemental/ 10.1103/PhysRevE.99.062602 for additional information and videos.

[58] V. Prymidis, H. Sielcken, and L. Filion, Soft Matter 11, 4158 (2015).

[59] W. L. Miller and A. Cacciuto, Phys. Rev. E 80, 021404 (2009).

[60] M. Pu, H. Jiang, and Z. Hou, Soft Matter 13, 4112 (2017). 